\title{
Uncertainties in field-line tracing in the magnetosphere. Part I: the axisymmetric part of the internal geomagnetic field
}

\author{
D. M. Willis ${ }^{1, *}$, J. Robin Singh ${ }^{1,2}$, Jacqueline Comer $^{1,2}$ \\ 1 Rutherford Appleton Laboratory, Chilton, Didcot, Oxon OX11 0QX, UK \\ 2 School of Mathematical and Information Sciences, Coventry University, Coventry, CV1 5FB, UK
}

Received: 6 November 1995 / Revised: 30 May 1996 / Accepted: 11 September 1996

\begin{abstract}
The technique of tracing along magnetic field lines is widely used in magnetospheric physics to provide a "magnetic frame of reference" that facilitates both the planning of experiments and the interpretation of observations. The precision of any such magnetic frame of reference depends critically on the accurate representation of the various sources of magnetic field in the magnetosphere. In order to consider this important problem systematically, a study is initiated to estimate first the uncertainties in magnetic-field-line tracing in the magnetosphere that arise solely from the published (standard) errors in the specification of the geomagnetic field of internal origin. Because of the complexity in computing these uncertainties for the complete geomagnetic field of internal origin, attention is focused in this preliminary paper on the uncertainties in magnetic-fieldline tracing that result from the standard errors in just the axisymmetric part of the internal geomagnetic field. An exact analytic equation exists for the magnetic field lines of an arbitrary linear combination of axisymmetric multipoles. This equation is used to derive numerical estimates of the uncertainties in magnetic-field-line tracing that are due to the published standard errors in the axisymmetric spherical harmonic coefficients (i.e. $\left.g_{n}^{0} \pm \delta g_{n}^{0}\right)$. Numerical results determined from the analytic equation are compared with computational results based on stepwise numerical integration along magnetic field lines. Excellent agreement is obtained between the analytical and computational methods in the axisymmetric case, which provides great confidence in the accuracy of the computer program used for stepwise numerical integration along magnetic field lines. This computer program is then used in the following paper to estimate the uncertainties in magnetic-field-line tracing in the magnetosphere that arise from the published standard errors in the full set of
\end{abstract}

\footnotetext{
* Also Visiting Reader in Physics, University of Sussex, Falmer, Brighton, BN1 9QH, UK

Correspondence to: D. M. Willis
}

spherical harmonic coefficients, which define the complete (non-axisymmetric) geomagnetic field of internal origin. Numerical estimates of the uncertainties in magnetic-field-line tracing in the magnetosphere, calculated here for the axisymmetric part of the internal geomagnetic field, should be regarded as "first approximations" in the sense that such estimates are only as accurate as the published standard errors in the set of axisymmetric spherical harmonic coefficients. However, all procedures developed in this preliminary paper can be applied to the derivation of more realistic estimates of the uncertainties in magnetic-field-line tracing in the magnetosphere, following further progress in the determination of more accurate standard errors in the spherical harmonic coefficients.

\section{Introduction}

The technique of tracing along magnetic field lines is widely used in magnetospheric physics to provide a "magnetic frame of reference" that facilitates both the planning of experiments and the interpretation of observations. More generally, the Earth's magnetic field provides a basic coordinate system for studying the distribution and movement of plasmas and energetic charged particles in the ionosphere and magnetosphere (Chapman and Bartels, 1940; Mcllwain, 1961; Akasofu and Chapman, 1972; Stern, 1976, 1994; Stern and Tsyganenko, 1992). For example, precise knowledge of the geomagnetic field is important in detailed studies of: (i) the motion of trapped particles that form the "Van Allen radiation belts" (Roederer, 1972; Walt, 1994); (ii) the precipitation of auroral particles into the upper atmosphere (McIlwain, 1960; Albert, 1967; Evans, 1968; Eather, 1973; Meng, 1978; Feldstein and Galperin, 1985; Gorney, 1987; Newell et al., 1991); and (iii) the trajectories of energetic solar protons and galactic cosmic rays in the vicinity of the Earth (Störmer, 1955; 
Vallarta, 1961; Alfvén and Fälthammar, 1963; Northrop, 1963; Roederer, 1970; Baker et al., 1990; Shea and Smart, 1990). In many branches of solar-terrestrial physics, the International Geomagnetic Reference Field (IGRF), or Definitive Geomagnetic Reference Field (DGRF), which specifies the (external) magnetic field originating from electric currents within the interior of the Earth (Peddie, 1982; Langel, 1992), provides a fundamental magnetic reference system (Hultqvist, 1958, McIlwain, 1961; Hakura, 1965; Gustafsson, 1970, 1984; Stassinopoulos et al., 1984; Baker and Wing, 1989; Gustafsson et al., 1992; Papitashvili et al., 1992). This reference magnetic field is also used extensively in the analysis and interpretation of ionospheric plasma velocities measured by both coherentand incoherent-scatter radars (Greenwald et al., 1978; Nielson et al., 1983; Rishbeth and Williams, 1985; Baker et al., 1989). Quite apart from its importance in solarterrestrial physics, the IGRF provides a basic reference frame, or coordinate system, that is used in air and marine navigation, exploration geophysics, land surveying and the drilling of oil wells (Stuart et al., 1988; Barraclough, 1990; Meyers and Davis, 1990).

The magnetic field in the magnetosphere arises from six main sources: (1) currents flowing in the Earth's liquid metallic (electrically conducting) outer core; (2) ionospheric currents; (3) field-aligned (or Birkeland) currents; (4) ring currents; (5) magnetopause currents; and (6) magnetotail currents (Stern and Tsyganenko, 1992). Any realistic model of the magnetic field in the magnetosphere must include accurate representations of each source. In practice, such models are usually modular, being based on the superposition of a subset of the six separate sources. Some of these sources are represented more accurately and realistically than others. For example, magnetic fields resulting from currents in the Earth's core, ring currents and magnetotail currents are reasonably realistic (in decreasing order of accuracy), whereas magnetic fields arising from ionospheric currents, field-aligned currents and magnetopause currents are usually represented by an allpurpose "polynomial" rather than separate modules (Stern and Tsyganenko, 1992). Magnetospheric models also need to be sufficiently flexible to allow for various known factors that affect the magnetosphere, such as the tilt angle $(\Psi)$, the complement of the angle between the geomagnetic (dipole) axis and the Sun-Earth line. Other factors that influence the state of the magnetosphere are represented quantitatively by indices such as the auroral electrojet index (AE), the equatorial ring current index (Dst), the solar-wind pressure (p), the interplanetary magnetic field (IMF) and the area of the polar cap.

The uses and limitations of magnetic-field models, as well as the concomitant uses and abuses of magneticfield-line tracing in the magnetosphere, have been discussed by several authors (e.g. Tsyganenko, 1987, 1989, 1990, 1991, 1993; Birn et al., 1991; Elphinstone et al., 1991; Fairfield, 1991; Pulkkinen, 1991; Pulkkinen et al., 1991, 1992; Stasiewicz, 1991; Stern and Tsyganenko, 1992; Baker et al., 1993; Stern, 1990, 1993, 1994;
Jordan, 1994). Although the applicability and utility of the various magnetic-field models are contentious matters, there can be no doubt that an accurate model of the magnetic field in the magnetosphere would be of great scientific value. This paper begins a systematic study of the uncertainties in field-line tracing in the magnetosphere by considering first those uncertainties that arise solely from possible errors in the specification of the geomagnetic field of internal origin (i.e. DGRF or IGRF). Characteristic uncertainties in field-line tracing in the magnetosphere are estimated using one of the few published models of the geomagnetic field that presents both the spherical harmonic coefficients and their standard errors (Langel et al., 1989, 1992). Uncertainties in the specification of the external sources of magnetic field in the magnetosphere (viz. ionospheric, Birkeland, ring, magnetopause and magnetotail currents) may produce comparable, or even larger, uncertainties in field-line tracing. However, it must be emphasized that the purpose of this and the following paper is to initiate a systematic study of the possible errors in field-line tracing in the magnetosphere, starting with the geomagnetic field of internal origin (DGRF or IGRF).

The motivation for this study stems largely from the frequent requirement in solar-terrestrial physics to compare measurements made by ground-based and satellite-borne instruments. In the past, such comparisons have often employed the technique of tracing along magnetic field lines in the magnetosphere without providing any estimate of the associated uncertainties. In this and the following companion paper, an effort is made to determine the characteristic uncertainties in magnetic-field-line tracing that result from the inevitable uncertainties in the specification of the Earth's internal magnetic field. In particular, estimates are presented for the uncertainty in tracing along a magnetic field line from the surface of the Earth to either the magnetic equatorial plane or the magnetic conjugate point. Although the present pair of papers merely report the first results of an ongoing systematic study of all possible sources of uncertainty in magnetic-field-line tracing in the magnetosphere, it is hoped that these initial results will facilitate certain comparisons between measurements made on the ground and in space.

\section{The main magnetic field of the Earth}

It will be shown that a detailed analytic discussion of the uncertainties in magnetic-field-line tracing in the magnetosphere is only practicable for the axisymmetric part of the Earth's main magnetic field. Nevertheless, it is convenient to formulate the problem in terms of the general spherical harmonic expansion of the geomagnetic field of internal origin. This approach not only provides a sound framework for subsequent numerical calculations of the uncertainty in field-line tracing for the complete geomagnetic field, but also serves to illustrate the very large number of field-line integrations required. 


\subsection{Spherical harmonic analysis of the main field}

At any given instant of time (epoch) the external scalar potential $V(r, \theta, \phi)$ of the Earth's main magnetic field, which is of internal origin, can be expressed in the form (Chapman and Bartels, 1940; Roederer, 1972; Stern, 1976, 1994; Langel, 1992)

$$
\begin{aligned}
V(r, \theta, \phi)= & R_{\mathrm{E}} \sum_{n=1}^{\infty}\left(R_{\mathrm{E}} / r\right)^{n+1} \\
& \times \sum_{m=0}^{n}\left(g_{n}^{m} \cos m \phi+h_{n}^{m} \sin m \phi\right) P_{n}^{m}(\cos \theta) .
\end{aligned}
$$

This spherical harmonic expansion, which is obtained by solving Laplace's equation $\left(\nabla^{2} V=0\right)$, is valid only outside the region of origin of the Earth's main magnetic field (predominantly the liquid metallic outer core), in an ideal external region containing no sources of magnetic field (i.e. curl $B=0$ ). In this particular representation of the geomagnetic field, positions on the surface of the Earth are specified in terms of geocentric spherical polar coordinates $(r, \theta, \phi)$ with origin $\mathrm{O}$ at the centre of the Earth; $r$ is the geocentric radial distance in $\mathrm{km}\left(r \geq R_{\mathrm{E}}\right), \theta$ is the geographic colatitude with the north geographic pole at $\theta=0$, and $\phi$ is the geographic longitude measured east from Greenwich. The radius of the reference sphere, $r=R_{\mathrm{E}}$, is taken to be the mean radius of the Earth $(6371.2 \mathrm{~km})$; $P_{n}^{m}(\cos \theta)$ is Schmidt's partially (or quasi-) normalized associated Legendre function of order $m$ and degree $n$ (where $m$ and $n$ are integers; $m \in \mathbb{I}_{0}^{+}$and $\left.n \in \mathbb{I}^{+}\right) ; g_{n}^{m}$ and $h_{n}^{m}$ are the spherical harmonic (or Schmidt) coefficients for the particular epoch considered, expressed in units of nanotesla (nT); and all physical quantities are measured in SI units. In this paper the definition of scalar magnetic potential $(V)$ is such that the spherical harmonic coefficients $g_{n}^{m}$ and $h_{n}^{m}$ have the (conventional) dimensions of magnetic induction (i.e. $\boldsymbol{B}=-\operatorname{grad} V)$.

\subsection{Determination of the spherical harmonic coefficients}

The numerical values of the spherical harmonic coefficients $g_{n}^{m}$ and $h_{n}^{m}$, which occur in Eq. 1, are usually calculated from a weighted-least-squares fitting procedure that uses all available magnetic data (for a particular epoch) on a global scale, from observatories, satellites and repeat stations, as well as from various aeromagnetic, shipborne and land surveys (Langel et al., 1989, 1992; Barraclough, 1990). To perform the least-squares analysis, the infinite summation in Eq. 1 is replaced by a finite summation that terminates at degree $n_{\max }$ (i.e. $1 \leq n \leq n_{\max }$, rather than $\left.1 \leq n<\infty\right)$. In theory, Eq. 1 is valid only if $n_{\max }$ is infinitely large but, in practice, $n_{\max }$ is restricted to a finite value by the inability of the data to resolve spherical harmonic coefficients of degree greater than $n_{\max }$ (Langel, 1992). For most epochs, the available magnetic data do not justify a value of $n_{\max }$ greater than
10. This value is kept constant in the various DGRF and IGRF models to maintain consistency.

It is known from magnetic measurements at the surface of the Earth that the geomagnetic field changes gradually with time (Chapman and Bartels, 1940; Langel, 1992). Thus the spherical harmonic coefficients $g_{n}^{m}$ and $h_{n}^{m}$ are really functions of time $(t)$. It is assumed that these coefficients vary linearly with time according to the equation (Langel et al., 1992)

$g(t)=g\left(t_{0}\right)+\int_{t_{0}}^{t} \dot{g}(\tau) d \tau$

where the dot denotes differentiation with respect to time. A particular geomagnetic reference field (DGRF or IGRF) comprises sets of tabulated values of the spherical harmonic coefficients $g_{n}^{m}$ and $h_{n}^{m}$, which define the main-field models at epochs separated by five years. In addition, a predictive secular-variation model defined by $\dot{g}_{n}^{m}$ and $\dot{h}_{n}^{m}$, expressed in units of nT/year, is used to extrapolate the geomagnetic reference field up to five years beyond the epoch of the latest main-field model. The maximum degree $\left(n_{\max }\right)$ of this secular-variation model is usually 8 . For any date prior to the latest epoch, which does not coincide with an epoch of one of the quinquennial main-field models, linear interpolation between the two main-field models that delimit the specified date is employed to derive actual magnetic-field components.

\subsection{Uncertainties in the spherical harmonic coefficients}

Since the spherical harmonic coefficients $g_{n}^{m}\left(t_{0}\right)$ and $h_{n}^{m}\left(t_{0}\right)$, for a particular epoch $t_{0}$, are calculated using a weighted-least-squares fitting procedure, there are uncertainties ("error bars") associated with the numerical values of these coefficients (Barraclough, 1990; Lowes, 1990a,b). A few published models of the geomagnetic field [viz. the NASA Goddard Space Flight Center Models: GSFC (12/83); GSFC 1985S; GSFC 1985DS; GSFC 1990S; GSFC 1990DS] give numerical values for both the spherical harmonic coefficients and their standard errors (Langel et al., 1989, 1992). The standard error in each spherical harmonic coefficient is approximately comparable with the magnitude of the corresponding annual variation, at least for the candidate models for DGRF 1985 and IGRF 1990 (Barraclough et al., 1992; Langel et al., 1992).

Although some earlier spherical harmonic models of the geomagnetic field quoted uncertainty values for the model coefficients, these were, without exception, based simply on how well the model fitted the data, and did not take account of any correlations between coefficients. Consequently, these earlier uncertainties underestimated the actual uncertainties by a factor of between 2 and 10. The model published by Langel et al. (1989) represents the first attempt to estimate realistic coefficient uncertainties. In their model, which is essentially a revision of an earlier model, approximate allowance is made for the magnetic field arising from neglected 
higher-degree fields (truncation), the presence of crustal magnetic fields and the existence of external magnetic fields, particularly $\mathrm{S}_{\mathrm{q}}$ (which represents the solar diurnal variation of ionospheric currents under quiet geomagnetic conditions). Subsequently, Langel et al. (1992) presented further models, which are the first to be published anew with realistic uncertainty values. These models also take account of neglected higher-degree fields (truncation), crustal fields and $\mathrm{S}_{\mathrm{q}}$. It is believed that the resulting (standard) error estimates are a good indicator of actual coefficient accuracy (Langel et al., 1992), at least to within a factor of 2 (Langel et al., 1989). Hopefully, future research will result in even more realistic estimates of the errors in the spherical harmonic coefficients, based on a more rigorous treatment of both systematic and stochastic errors.

The uncertainties ("error bars") associated with the spherical harmonic coefficients $g_{n}^{m}$ and $h_{n}^{m}$ are denoted in this paper by $\delta g_{n}^{m}$ and $\delta h_{n}^{m}$, respectively. In illustrative numerical calculations, $\delta g_{n}^{m}$ and $\delta h_{n}^{m}$ are assumed to be multiples of the corresponding standard errors, although the procedures developed here are equally valid for other realistic numerical estimates of $\delta g_{n}^{m}$ and $\delta h_{n}^{m}$. Any discussion of the accuracy of the geomagnetic field, or the accuracy of field-line tracing in the magnetosphere, must therefore allow for the fact that the numerical value of each spherical harmonic coefficient $g_{n}^{m}$, or $h_{n}^{m}$, is not exact but lies (in a statistical sense) within the interval $\left[g_{n}^{m}-\delta g_{n}^{m}\right.$, $\left.g_{n}^{m}+\delta g_{n}^{m}\right]$, or $\left[h_{n}^{m}-\delta h_{n}^{m}, h_{n}^{m}+\delta h_{n}^{m}\right]$, where $\delta g_{n}^{m} \geq 0$ and $\delta h_{n}^{m} \geq 0$.

\subsection{Range of a geomagnetic-field model}

The determination of the uncertainty in the accuracy of field-line tracing in the magnetosphere is now conceptually equivalent to a mathematical problem in (multiple) interval analysis. Each spherical harmonic coefficient $g_{n}^{m}$ is assumed to lie in the interval of real numbers $\left[g_{n}^{m}-\delta g_{n}^{m}, g_{n}^{m}+\delta g_{n}^{m}\right]$, which is represented by the ordered pair of real numbers (the "lower" and "upper" end-points), $g_{n}^{m}-\delta g_{n}^{m}$ and $g_{n}^{m}+\delta g_{n}^{m}\left(\delta g_{n}^{m} \geq 0\right)$; similarly for $h_{n}^{m}$. The "range", or variability, of a geomagnetic-field model (DGRF or IGRF) at any epoch $t_{0}$ is defined in terms of the set of "limiting geomagnetic-field models", which results from selecting every possible permutation of the pairs of "end-points" for $g_{n}^{m}$ and $h_{n}^{m}$. If the spherical harmonic expansion defined by Eq. 1 is truncated at degree $n_{\max }$, the number $(N)$ of spherical harmonic coefficients is given by $N=n_{\max }\left(n_{\max }+2\right)$ in the general case. Hence the number $(\mathscr{N})$ of "limiting geomagnetic-field models" $(\mathscr{G})$ is given by $\mathscr{N}=2^{N}$ and the set of such field models can be expressed symbolically in the form $\left\{\mathscr{G}_{1}, \mathscr{G}_{2}\right.$, $\left.\mathscr{G}_{3}, \ldots, \mathscr{G}_{\mathcal{N}}\right\}$. Since it is necessary to refer this variability to the mean (or central) geomagnetic-field model $\mathscr{G}_{0}$ (which is defined by the set of central values $g_{n}^{m}$ and $h_{n}^{m}$ ), the number of different "models" to be considered in this method of determining the uncertainty in field-line tracing is $\mathscr{N}+1$, that is $2^{N}+1$. This approach involves considering all possible permutations of the "endpoints".

The uncertainty in the geocentric distance $(r)$ of an arbitrary point on a magnetic field line is denoted symbolically by $\delta r$, where $\delta r \in \mathbb{R}$. The mean (or central) model $\mathscr{G}_{0}$ corresponds to $r$, whereas the models with errors associated with them, namely $\left\{\mathscr{G}_{1}, \mathscr{G}_{2}\right.$, $\left.\mathscr{G}_{3}, \ldots, \mathscr{G}_{\mathcal{N}}\right\}$, correspond to a set of values $\{r+\delta r\}$. For just the axisymmetric part of the geomagnetic field (with which this paper is largely concerned) the set of values $\{r+\delta r\}$, corresponding to the set of geomagnetic field lines passing through a fixed "starting" point (for example, a point on the surface of the Earth), lie in a meridian plane. In principle, $\delta r$ can be measured uniquely with respect to $r$ in any convenient direction in the meridian plane through this fixed "starting point". For the general (non-axisymmetric) geomagnetic field, however, the set of values $\{r+\delta r\}$ does not lie in a meridian plane and $\delta r$ must then be regarded as the magnitude $(|\boldsymbol{\delta} \boldsymbol{r}|)$ of a vector $\boldsymbol{\delta} \boldsymbol{r}$, whose strict definition also involves an azimuthal angle with respect to the central vector $\boldsymbol{r}$ (as discussed in greater detail in the following companion paper). In practice, an important goal in the following analysis is to find the largest possible value for $\delta r\left(\delta r_{\max }\right)$ at the geomagnetic equator, or on the surface of the Earth in the opposite hemisphere, for any given starting point on the surface of the Earth. The field model $\mathscr{G}_{\max }$ that gives the maximum value $\delta r_{\max }$ clearly belongs to the set of $\mathscr{N}$ limiting geomagnetic-field models.

Table 1 indicates that, in the general (non-axisymmetric) case, the number of limiting geomagnetic-field models increases very rapidly as the highest degree $\left(n_{\max }\right)$ increases to only a modest value $\left(n_{\max }=5\right)$. For example, if a computer program were used to trace (by stepwise numerical integration) along a geomagnetic field line, starting at a fixed point on the Earth's surface, more than 16.7 million field-line tracings would be required to determine the uncertainty in the location of the point where this particular field line crosses the geomagnetic equator (say) in the case $n_{\max }=4$.

Owing to the extremely large number of field-line tracings required in the general case $\left(g_{n}^{m}, h_{n}^{m} ; 0 \leq m \leq n\right.$, $\left.1 \leq n \leq n_{\max }\right)$, the uncertainties in field-line tracing in the magnetosphere are first investigated analytically for just the axisymmetric part $(m=0)$ of the internal geomagnetic field $\left(g_{n}^{0} ; 1 \leq n \leq n_{\max }\right)$. In the special case $m=0$, the number $(N)$ of spherical harmonic coefficients is given by $N=n_{\max }$. The analytic approach in the

Table 1. The number of limiting geomagnetic-field models

\begin{tabular}{llr}
\hline $\begin{array}{l}\text { Highest } \\
\text { degree } \\
{\left[n_{\max }\right]}\end{array}$ & $\begin{array}{l}\text { Number of } \\
\text { coefficients } \\
{\left[N=n_{\max }\left(n_{\max }+2\right)\right]}\end{array}$ & $\begin{array}{l}\text { Number of } \\
\text { limiting models } \\
{\left[\mathcal{N}=2^{N}\right]}\end{array}$ \\
\hline 1 & 3 & 8 \\
2 & 8 & 256 \\
3 & 15 & 32768 \\
4 & 24 & 16777216 \\
5 & 35 & 34359738368 \\
\hline
\end{tabular}


axisymmetric case provides deeper physical insight into this complicated problem and also provides a method of checking the numerical accuracy of a computer program that has been developed to trace along magnetic field lines by stepwise numerical integration. Once the accuracy of this computer program has been validated in the general axisymmetric case $\left(n_{\max }=10\right)$, and a special axisymmetric case $\left(n_{\max }=4\right)$ has been examined analytically, the uncertainties in field-line tracing in the general non-axisymmetric case $\left(g_{n}^{m}, h_{n}^{m} ; 0 \leq m \leq n\right.$, $1 \leq n \leq n_{\max }$ ) are evaluated in the following paper by Willis et al. (1997), hereafter referred to as Paper II.

\section{The axisymmetric part of the geomagnetic field}

An exact equation for the magnetic field lines of an arbitrary axisymmetric magnetic field is presented in Sect. 3.1. In general, this equation cannot be solved analytically to express $r$ as a function of $\theta$. However, it is shown in Sect. 3.2 that in the special case $n_{\max }=4$, corresponding to the summation of the axially symmetric dipole $\left(g_{1}^{0}\right)$, quadrupole $\left(g_{2}^{0}\right)$, octupole $\left(g_{3}^{0}\right)$ and sedecimupole $\left(g_{4}^{0}\right)$ terms, this exact equation reduces to a quartic, which can be solved analytically. [Some authors use the term "hexadecapole" rather than "sedecimupole"; the latter term was introduced by Winch (1967).] In the magnetic equatorial plane, the quartic simplifies still further to a cubic. The sign of the discriminant of this cubic equation depends on both the magnitude and sign of the ratio $g_{3}^{0} / g_{1}^{0}$.

\subsection{General axisymmetric case}

Backus (1988) has derived an exact equation for the magnetic field lines of an arbitrary axisymmetric magnetic field $\boldsymbol{B}$ outside a sphere $S\left(R_{\mathrm{E}}\right)$, of radius $R_{\mathrm{E}}$, containing all sources of $\boldsymbol{B}$. Using the notation adopted in this paper, his equation for the magnetic field lines can be expressed in the form (cf. his Eq. 8)

$r^{2} \sin ^{2} \theta \sum_{n=1}^{\infty} n^{-1} g_{n}^{0}\left(R_{\mathrm{E}} / r\right)^{n+2} P_{n}^{\prime}(\cos \theta)=K$,

where the prime denotes differentiation with respect to $\cos \theta$ and $K$ denotes a constant. Making the substitution $\mu=\cos \theta$, so that $d \mu / d \theta=-\sin \theta$ and $P_{n}^{\prime}(\mu)=d P_{n}^{0}(\mu) / d \mu$, it can be seen immediately that $P_{n}^{\prime}(\mu)=-\left[d P_{n}^{0}(\cos \theta) / d \theta\right] / \sin \theta$. Moreover, since $d P_{n}^{0}(\cos \theta) / d \theta=-[n(n+1) / 2]^{1 / 2} P_{n}^{1}(\cos \theta)$ (Chapman and Bartels, 1940; Chap. XVII, Eq. 55), Eq. 3 can be written in the form

$R_{\mathrm{E}}^{2} \sum_{n=1}^{\infty}[(n+1) / 2 n]^{1 / 2} g_{n}^{0}\left(R_{\mathrm{E}} / r\right)^{n} \sin \theta P_{n}^{1}(\cos \theta)=K$.

Equation 4 is an exact analytic expression for the magnetic field lines resulting from the axisymmetric scalar magnetic potential defined by Eq. 1 with $m=0\left(h_{n}^{0} \equiv 0\right)$.
If all spherical harmonic coefficients in the above summation are zero apart from the first (i.e. $g_{1}^{0} \neq 0, g_{n}^{0}=0$ for $n \geq 2$ ), Eq. 4 reduces to the form $R_{\mathrm{E}}^{2} g_{1}^{0}\left(R_{\mathrm{E}} / r\right) \sin ^{2} \theta=K$, since $P_{1}^{1}(\cos \theta)=\sin \theta$. Assuming that $r=r_{1}$ when $\theta=\pi / 2$, this simple equation becomes

$r=r_{1} \sin ^{2} \theta$,

which is the well-known equation for the field lines of a magnetic dipole aligned with the polar axis $(\theta=0, \pi)$. The parameter $r_{1}\left(=R_{\mathrm{E}} / \sin ^{2} \theta_{0}\right)$ specifies the geocentric distance at which a dipole magnetic field line, which intersects the reference sphere, $r=R_{\mathrm{E}}$, at colatitude $\theta_{0}$, crosses the equatorial plane $(\theta=\pi / 2)$. This last equation indicates that the magnetic-field configuration of a dipole is independent of the dipole strength $\left(g_{1}^{0}\right)$.

Similarly, if all terms in the summation vanish apart from the $n^{\text {th }}$, Eq. 4 simplifies to the form $R_{\mathrm{E}}{ }^{2}[(n+1) / 2 n]^{1 / 2} g_{n}^{0}\left(R_{\mathrm{E}} / r\right)^{n} \sin \theta P_{n}^{1}(\cos \theta)=K$. If the constant $K$ is taken to be $R_{\mathrm{E}}^{2}[(n+1) / 2 n]^{1 / 2} g_{n}^{0}\left(R_{\mathrm{E}} / r_{n}\right)^{n}$, where the parameter $r_{n}$ defines (not necessarily directly) the geocentric distance of a magnetic field line, this last equation becomes

$r=r_{n}\left[\left|\sin \theta P_{n}^{1}(\cos \theta)\right|\right]^{1 / n}$,

which is just the equation for the magnetic field lines of an individual axisymmetric magnetic multipole of degree $n$ (Willis and Young, 1987; cf. their Eq. 19).

Equation 4 can be rewritten in the more appropriate form

$$
\begin{aligned}
g_{1}^{0}\left(R_{\mathrm{E}}{ }^{3} / r\right) \sin ^{2} \theta & \llbracket 1+\sum_{n=2}^{\infty}[(n+1) / 2 n]^{1 / 2}\left(g_{n}^{0} / g_{1}^{0}\right) \\
& \times\left(R_{\mathrm{E}} / r\right)^{n-1} \quad P_{n}{ }^{1}(\cos \theta) / \sin \theta \rrbracket=K,(7)
\end{aligned}
$$

which is particularly useful if the dipole term $\left(g_{1}^{0}\right)$ predominates - as is the case for the contemporary geomagnetic field. The constant in Eq. 7 can be specified by assuming that a magnetic field line crosses the reference sphere, $r=R_{\mathrm{E}}$, at colatitude $\theta=\theta_{0}$. Then the equation for magnetic field lines in the axisymmetric case $(m=0)$ becomes

$r=r_{1} \sin ^{2} \theta \frac{\llbracket\left[1+\sum_{n=2}^{\infty}[(n+1) / 2 n]^{1 / 2}\left(g_{n}^{0} / g_{1}^{0}\right)\left(R_{\mathrm{E}} / r\right)^{n-1} P_{n}^{1}(\cos \theta) / \sin \theta\right]}{\left.\llbracket\left[1+\sum_{n=2}^{\infty}[(n+1) / 2 n]^{1 / 2}\left(g_{n}^{0} / g_{1}^{0}\right) P_{n}^{1}\left(\cos \theta_{0}\right) / \sin \theta_{0}\right]\right]}$.

Here $r_{1}\left(=R_{\mathrm{E}} / \sin ^{2} \theta_{0}\right)$ again denotes the geocentric distance at which a dipole magnetic field line crosses the equatorial plane $(\theta=\pi / 2)$. [In the axisymmetric case $(m=0)$, the geographic and geomagnetic equators coincide.] Equation 8 reduces correctly to Eq. 5 if only the dipole term $(n=1)$ is present (i.e. $g_{1}^{0} \neq 0, g_{n}^{0}=0$ for $\left.n \geq 2\right)$. It is important to emphasize that Eq. 8 refers to a magnetic field line (or, more correctly, a shell of magnetic field lines) passing through the fixed point (or azimuthal ring of points) $r=R_{\mathrm{E}}, \theta=\theta_{0}(0 \leq \phi \leq 2 \pi)$, irrespective of the actual values of the spherical harmonic coefficients $g_{n}^{0}$. 
Stated simply, the shell of magnetic field lines is deliberately anchored at colatitude $\theta=\theta_{0}$ on the (mean) surface of the Earth $\left(r=R_{\mathrm{E}}\right)$. This choice of constant in Eq. 7 facilitates comparisons with computer programs that trace along magnetic field lines by stepwise numerical integration, starting at a fixed point on the Earth's surface (see Sect. 4.4).

In the axisymmetric case $(m=0)$, it is particularly convenient to consider the uncertainty in the position of the point where a field line crosses the (magnetic) equatorial plane. It follows from Eq. 8 that the geocentric distance of a field line in the equatorial plane $(\theta=\pi / 2)$ is given by

$$
r=r_{1} \frac{\llbracket 1+\sum_{n=2}^{\infty}[(n+1) / 2 n]^{1 / 2}\left(g_{n}^{0} / g_{1}^{0}\right)\left(R_{\mathrm{E}} / r\right)^{n-1} P_{n}^{1}(0) \rrbracket}{\llbracket 1+\sum_{n=2}^{\infty}[(n+1) / 2 n]^{1 / 2}\left(g_{n}^{0} / g_{1}^{0}\right) P_{n}^{1}\left(\cos \theta_{0}\right) / \sin \theta_{0} \rrbracket} .
$$

Moreover, Schmidt's partially normalized associated Legendre function $P_{n}^{1}(0)$ can be expressed in the following form (Erdélyi et al., 1953; Vol. 1, Sect. 3.4, amended Eq. 20)

$$
\begin{aligned}
P_{n}^{1}(0)= & -2[2 / n(n+1)]^{1 / 2} \pi^{-1 / 2} \cos [(n+1) \pi / 2] \\
& \times \Gamma(n / 2+1) / \Gamma(n / 2+1 / 2),
\end{aligned}
$$

where $\Gamma$ denotes the Gamma function. The presence of the trigonometric term in Eq. 10 implies that

and

$$
\left.\begin{array}{l}
P_{n}^{1}(0) \neq 0 \text { if } n \text { is odd } \\
P_{n}^{1}(0)=0 \text { if } n \text { is even }
\end{array}\right\} \text {. }
$$

Therefore, it is only the axially symmetric multipoles $\left(g_{n}^{0}\right)$ of odd degree $(n)$ that actually contribute to the numerator in Eq. 9, although multipoles of both odd and even degree contribute to the "normalization factor" in the denominator. This property of $P_{n}^{1}(0)$ significantly reduces the number of terms in the polynomial equation of degree $n_{\max }\left(n_{\max }\right.$ odd) or $n_{\max }-1$ ( $n_{\max }$ even), which defines the geocentric distance of field lines in the equatorial plane if the infinite summations in Eq. 9 are truncated at degree $n_{\max }$. This reduction in the number of terms simplifies the subsequent analysis.

\subsection{Special axisymmetric case $\mathrm{n}_{\max }=4$}

The equations derived in the previous subsection are completely general. In particular, Eq. 8 provides an exact analytic expression for the field lines of the axisymmetric magnetic field obtained by ignoring the non-axisymmetric part $(m \neq 0)$ of the main geomagnetic field defined by Eq. 1. Nevertheless, Eq. 8 cannot be solved analytically to express $r$ as a function of $\theta$ in the general case. It is shown in this subsection, however, that $r$ can be expressed explicitly in terms of $\theta$ for the axisymmetric magnetic field arising from the sum- mation of just the first four (axisymmetric) multipoles $\left(n_{\max }=4\right)$; namely, a dipole $\left(g_{1}^{0}\right)$, a quadrupole $\left(g_{2}^{0}\right)$, an octupole $\left(g_{3}^{0}\right)$ and a sedecimupole $\left(g_{4}^{0}\right)$.

If the infinite summations in Eq. 8 are restricted to finite summations with $n_{\max }=4$, and the associated Legendre functions are replaced by their trigonometric forms (Matsushita and Campbell, 1967), the equation for magnetic field lines reduces to the form

$$
\begin{aligned}
r= & r_{1} \sin ^{2} \theta\left[1+(3 / 2)\left(g_{2}^{0} / g_{1}^{0}\right)\left(R_{\mathrm{E}} / r\right) \cos \theta\right. \\
& +(1 / 2)\left(g_{3}^{0} / g_{1}^{0}\right)\left(R_{\mathrm{E}} / r\right)^{2}\left(5 \cos ^{2} \theta-1\right) \\
& \left.+(5 / 8)\left(g_{4}^{0} / g_{1}^{0}\right)\left(R_{\mathrm{E}} / r\right)^{3} \cos \theta\left(7 \cos ^{2} \theta-3\right)\right] / F \\
& \times\left(\cos \theta_{0}\right),
\end{aligned}
$$

where

$$
\begin{aligned}
F\left(\cos \theta_{0}\right)= & 1+(3 / 2)\left(g_{2}^{0} / g_{1}^{0}\right) \cos \theta_{0} \\
& +(1 / 2)\left(g_{3}^{0} / g_{1}^{0}\right)\left(5 \cos ^{2} \theta_{0}-1\right) \\
& +(5 / 8)\left(g_{4}^{0} / g_{1}^{0}\right) \cos \theta_{0}\left(7 \cos ^{2} \theta_{0}-3\right) .
\end{aligned}
$$

Equation 12 is a quartic in $r$ that can be solved analytically to express $r$ as a function of $\theta$. Inclusion of the axisymmetric duotrigintupole $\left(g_{5}^{0}\right)$ results in a quintic equation that, in general, cannot be solved analytically (i.e. for arbitrary values of the spherical harmonic coefficients $\left.g_{n}^{0}, 1 \leq n \leq 5\right)$; similarly for higher-degree axisymmetric multipoles $(n>5)$.

The roots of Eq. 12 are extremely complicated and hence the following discussion is restricted to the equation for the point at which an individual magnetic field line crosses the equatorial plane $(\theta=\pi / 2)$, namely

$r^{3}-\left(r_{1} / F\right) r^{2}+(1 / 2)\left(g_{3}^{0} / g_{1}^{0}\right) R_{\mathrm{E}}^{2}\left(r_{1} / F\right)=0$,

where $F$ is used as an abbreviation for $F\left(\cos \theta_{0}\right)$, defined by Eq. 13. The form of Eq. 14 is entirely consistent with the earlier statement (cf. Sect. 3.1) that it is only the axisymmetric multipoles $\left(g_{n}^{0}\right)$ of odd degree $(n)$ that yield non-zero coefficients in the polynomial equation defining the geocentric distance at which a field line crosses the equatorial plane $(\theta=\pi / 2)$.

The discriminant, $D$, of the simpler cubic equation can be expressed in the form

$$
\begin{aligned}
D= & 2\left(g_{3}^{0} / g_{1}^{0}\right) R_{\mathrm{E}}^{2}\left(r_{1} / F\right)^{4} \\
& \times\left[1-(27 / 8)\left(g_{3}^{0} / g_{1}^{0}\right) \sin ^{4} \theta_{0} F^{2}\right]
\end{aligned}
$$

if the equation $R_{\mathrm{E}} / r_{1}=\sin ^{2} \theta_{0}$ is used to eliminate $\left(R_{\mathrm{E}} / r_{1}\right)^{2}$. Since $g_{3}^{0} / g_{1}^{0}<0$ for the contemporary geomagnetic field (Barraclough et al., 1992) and the timeaveraged palaeomagnetic field for both the Brunhes (normal) and Matuyama (reversed) epochs (Schneider and Kent, 1990), it follows from Eq. 15 that $D<0$ for several realistic geomagnetic applications. If $D<0$, Eq. 14 has only one real, and hence physically meaningful, positive root. In this case each magnetic field line crosses the equatorial plane only once $\left(r \geq R_{\mathrm{E}}\right)$.

It remains to consider the rather more conjectural cases for which $g_{3}^{0} / g_{1}^{0}>0$. This condition might 
possibly apply to the historical geomagnetic field before about 1700 AD (Barraclough, 1974; Thompson and Barraclough, 1982) and to certain idealized models of the transition magnetic field during a geomagnetic polarity reversal (Williams and Fuller, 1981; Weeks et al., 1988), provided the dipole term is not identically zero (i.e. $\left.g_{1}^{0} \neq 0\right)$ and any non-axisymmetric terms are small. If $g_{3}^{0} / g_{1}^{0}>0$ and $1<(27 / 8)\left(g_{3}^{0} / g_{1}^{0}\right) \sin ^{4} \theta_{0} F^{2}, D<0$ and once again there is only one physically meaningful root of Eq. 14, which implies that each magnetic field line crosses the equatorial plane only once $\left(r \geq R_{\mathrm{E}}\right)$. If $g_{3}^{0} / g_{1}^{0}>0$ and $1>(27 / 8)\left(g_{3}^{0} / g_{1}^{0}\right) \sin ^{4} \theta_{0} F^{2}, D>0$ and hence Eq. 14 has three real roots, although these roots are not all physically meaningful in the sense that each root is positive and greater than or equal to $R_{\mathrm{E}}$. It follows from Eq. 15 that the condition for multiple roots, $D=0$, is satisfied if $(27 / 8)\left(g_{3}^{0} / g_{1}^{0}\right) \sin ^{4} \theta_{0} F^{2}=1$.

\section{Uncertainties in field-line tracing for the axisymmetric part of the internal geomagnetic field}

The various analytical and computational methods of determining the uncertainties in magnetic-field-line tracing in the magnetosphere, resulting from just the axisymmetric part of the internal geomagnetic field, are described in this section. Attention is focused on the special case $n_{\max }=4$ and the general case $1 \leq n \leq n_{\max }$.

\subsection{Analytic solution in the special axisymmetric case $\mathrm{n}_{\max }=4$}

The geocentric distance at which an individual magnetic field line crosses the equatorial plane in the special axisymmetric case $n_{\max }=4$ is defined by Eq. 14. This cubic equation can be solved analytically either by using standard algebraic techniques (Jones, 1975) or by using the computer algebra package Mathematica (Wolfram, 1988). Both methods have been used to confirm that the three roots $(r)_{1},(r)_{2}$ and $(r)_{3}$ of Eq. 14 can be expressed in the exact form

$$
\begin{aligned}
(r)_{1}= & r_{1} / 3 F+\left[1+\left(r_{1} / 3 F \Re\right)^{2}\right] \Re, \\
(r)_{2}= & r_{1} / 3 F-(1 / 2)\left[1+\left(r_{1} / 3 F \Re\right)^{2}\right] \Re+i(\sqrt{3} / 2) \\
& \times\left[1-\left(r_{1} / 3 F \Re\right)^{2}\right] \Re, \\
(r)_{3}= & r_{1} / 3 F-(1 / 2)\left[1+\left(r_{1} / 3 F \Re\right)^{2}\right] \Re-i(\sqrt{3} / 2) \\
& \times\left[1-\left(r_{1} / 3 F \Re\right)^{2}\right] \Re,
\end{aligned}
$$

where $i=\sqrt{-1}, F$ is defined by Eq. 13 and

$$
\begin{aligned}
\Re= & \llbracket\left(r_{1} / 3 F\right)^{3}-\left(R_{\mathrm{E}} / 2\right)^{2}\left(r_{1} / F\right)\left(g_{3}^{0} / g_{1}^{0}\right) \\
& +\left\{\left[\left(r_{1} / 3 F\right)^{3}-\left(R_{\mathrm{E}} / 2\right)^{2}\left(r_{1} / F\right)\left(g_{3}^{0} / g_{1}^{0}\right)\right]^{2}\right. \\
& \left.-\left(r_{1} / 3 F\right)^{6}\right\}^{1 / 2} \rrbracket^{1 / 3} .
\end{aligned}
$$

The computer algebra package Mathematica has also been used to check that the three roots $(r)_{1},(r)_{2}$ and $(r)_{3}$, defined by Eqs. 16-19, actually satisfy Eq. 14 and the following conditions on the roots of this equation : $(r)_{1}+(r)_{2}+(r)_{3}=r_{1} / F ;(r)_{1}(r)_{2}+(r)_{2}(r)_{3}+(r)_{3}(r)_{1}$ $=0 ;$ and $(r)_{1}(r)_{2}(r)_{3}=-(1 / 2)\left(g_{3}^{0} / g_{1}^{0}\right) R_{\mathrm{E}}^{2}\left(r_{1} / F\right)$.

By inserting specific numerical values for $g_{n}^{0}(1 \leq n \leq 4), R_{\mathrm{E}}, r_{1}$ and $\theta_{0}$, Eqs. 16-19 provide the geocentric distance at which an individual magnetic field line crosses the equatorial plane in the special axisymmetric case $n_{\max }=4$. If Eq. 14 has three real roots, only one geocentric distance is physically meaningful in the sense that it is positive and greater than or equal to $R_{\mathrm{E}}$ (see Sect. 3.2). The parameters $\theta_{0}$ and $r_{1}$ are related by the equation for a dipole line of force, namely $R_{\mathrm{E}}=r_{1} \sin ^{2} \theta_{0}$. Therefore, $\theta_{0}$ and $r_{1}$ may be regarded as alternative labels that define a particular shell of magnetic field lines, as discussed in Sect. 3.1. Selecting a particular value of $\theta_{0}$ (or $r_{1}$ ) for the contemporary geomagnetic field, Eq. 16 can be used to find the real solutions of Eq. 14 for the mean (or central) values of the axisymmetric spherical harmonic coefficients $g_{n}^{0}(1 \leq n \leq 4)$ and for all $16\left(=2^{4}\right)$ permutations of the end points of the intervals $\left[g_{n}^{0}-\delta g_{n}^{0}, g_{n}^{0}+\delta g_{n}^{0}\right]$, as outlined in Sect. 2.4. This procedure provides 16 values of $\delta r$ from which the largest value, $\delta r_{\max }$, can be determined.

\subsection{Approximate analytic solution in \\ the general axisymmetric case $1 \leq n \leq n_{\max }$}

If the infinite summations in Eq. 9 are restricted to finite summations with $2 \leq n \leq n_{\max }$, the geocentric distance at which a magnetic field line crosses the equatorial plane becomes

$$
r=r_{1} \frac{\llbracket 1+\sum_{n=2}^{n_{\max }}[(n+1) / 2 n]^{1 / 2}\left(g_{n}^{0} / g_{1}^{0}\right)\left(R_{\mathrm{E}} / r\right)^{n-1} P_{n}^{1}(0) \rrbracket}{\llbracket 1+\sum_{n=2}^{n_{\max }}[(n+1) / 2 n]^{1 / 2}\left(g_{n}^{0} / g_{1}^{0}\right) P_{n}^{1}\left(\cos \theta_{0}\right) / \sin \theta_{0} \rrbracket} .
$$

As implied previously, this equation cannot be solved analytically in the general axisymmetric case $2 \leq n \leq n_{\max }$ to express $r$ explicitly as a function of $\theta_{0}$. However, it is possible to obtain an approximate solution of Eq. 20 if all terms under the summation signs are small compared with unity (i.e. if $\left|g_{n}^{0} / g_{1}^{0}\right| \ll 1$ for $2 \leq n \leq n_{\max }$ ). In this situation the binomial theorem can be used to obtain a power-series expansion of the small terms. Likewise, if allowance is made for the uncertainties $\delta g_{n}^{0}$ in the axisymmetric spherical harmonic coefficients $g_{n}^{0}\left(1 \leq n \leq n_{\max }\right)$, but triple and higher products of the small terms $\delta g_{n}^{0} / g_{n}^{0}\left(1 \leq n \leq n_{\max }\right)$ are neglected, an approximate expression can be derived for the uncertainty $(\delta r)$ in magnetic-field-line tracing in the magnetosphere.

Using this approach, it is shown in Appendix A that $\delta r$ can be expressed in the approximate form 


$$
\begin{aligned}
\delta r \approx & r_{1} \sum_{N^{*}=1}^{N_{\max }^{*}}(-1)^{N^{*}-1} \llbracket\left(N^{*}-1\right)\left(\sum_{n=2}^{n_{\max }} b_{n} G_{n}\right)^{N^{*}-3} \\
& \times\left(\sum_{n=2}^{n_{\max }} b_{n} \delta G_{n}\right)\left\{[ \sum _ { n = 2 } ^ { n _ { \operatorname { m a x } } } ( a _ { n } - b _ { n } ) G _ { n } ] \left[\left(\sum_{n=2}^{n_{\max }} b_{n} G_{n}\right)\right.\right. \\
& \left.+\frac{\left(N^{*}-2\right)}{2}\left(\sum_{n=2}^{n_{\max }} b_{n} \delta G_{n}\right)\right]+\left[\sum_{n=2}^{n_{\max }}\left(a_{n}-b_{n}\right) \delta G_{n}\right] \\
& \left.\times\left(\sum_{n=2}^{n_{\max }} b_{n} G_{n}\right)\right\}+\left(\sum_{n=2}^{n_{\max }} b_{n} G_{n}\right)^{N^{*}-1} \\
& \left.\times\left[\sum_{n=2}^{n_{\max }}\left(a_{n}-b_{n}\right) \delta G_{n}\right]\right],
\end{aligned}
$$

where, for $n \geq 2$,

$$
\begin{aligned}
G_{n}=g_{n}^{0} / g_{1}^{0}, \\
\begin{aligned}
\delta G_{n} / G_{n}= & \left(\delta g_{n}^{0} / g_{n}^{0}-\delta g_{1}^{0} / g_{1}^{0}\right) \\
& -\left(\delta g_{1}^{0} / g_{1}^{0}\right)\left(\delta g_{n}^{0} / g_{n}^{0}-\delta g_{1}^{0} / g_{1}^{0}\right),
\end{aligned}
\end{aligned}
$$

to second order in the small terms $\delta g_{1}^{0} / g_{1}^{0}$ and $\delta g_{n}^{0} / g_{n}^{0}$,

$a_{n}=[(n+1) / 2 n]^{1 / 2}\left(R_{\mathrm{E}} / r_{1}\right)^{n-1} P_{n}^{1}(0)$,

and

$b_{n}=[(n+1) / 2 n]^{1 / 2} P_{n}^{1}\left(\cos \theta_{0}\right) / \sin \theta_{0}$.

In Eq. 21 summations that really extend over the infinite range $1 \leq N^{*}<\infty$ are restricted to the finite range $1 \leq N^{*} \leq N_{\max }^{*}$, by analogy with the summations over the finite range $2 \leq n \leq n_{\max }$.

Contrary to the convention adopted in Sect. 2.3, $\delta g_{n}^{0}\left(1 \leq n \leq n_{\max }\right)$ can be either positive or negative in the set of equations just given (see Appendix A). Hence $\delta r$ can be either positive or negative in the case of the axisymmetric part $(m=0)$ of the internal geomagnetic field, as discussed further in Sect. 6.2. The set of values $\{r+\delta r\}$ defines a linear (or curvilinear) interval in which $\delta r$ is regarded as being positive or negative with respect to the central value $r$. If follows from Eq. 21 that $\delta r \rightarrow 0$ as $\delta G_{n} \rightarrow 0$ for $2 \leq n \leq n_{\max }$, which implies that if there are no uncertainties in the axisymmetric spherical harmonic coefficients $g_{n}^{0}\left(2 \leq n \leq n_{\max }\right)$ then there is no uncertainty in the geocentric distance $(r)$ at which a magnetic field line crosses the geomagnetic equator $(\theta=\pi / 2)$. This last result confirms the earlier conclusion that the magnetic-field configuration of a dipole is independent of the dipole strength $\left(g_{1}^{0}+\delta g_{1}^{0}\right)$. Finally, Eq. 21 reduces correctly to the case of a dipole plus an individual multipole of degree $n$ if the symbols denoting summation over $n$ are removed.

By analogy with the scheme discussed in Sect. 4.1, Eqs. 21-25 can be used to find the $2^{n_{\max }}$ values of $\delta r$ corresponding to the $2^{n_{\max }}$ permutations of the end points of the intervals $\left[g_{n}^{0}-\delta g_{n}^{0}, g_{n}^{0}+\delta g_{n}^{0}\right]$, from which the largest value, $\delta r_{\text {max }}$, can be determined.

\subsection{Computational solution in the general axisymmetric case $1 \leq n \leq n_{\max }$}

It follows from Eq. 20 that the geocentric distance at which a magnetic field line crosses the equatorial plane in the (truncated) axisymmetric case $1 \leq n \leq n_{\max }$ is given by

$$
\begin{aligned}
r^{n_{\max }}- & \left(r_{1} / F_{n_{\max }}\right) r^{n_{\max }-1}-(2 / 3)^{1 / 2}\left(g_{3}^{0} / g_{1}^{0}\right) \\
& \times R_{\mathrm{E}}^{2}\left(r_{1} / F_{n_{\max }}\right) P_{3}^{1}(0) r^{n_{\max }-3}-(3 / 5)^{1 / 2} \\
& \times\left(g_{5}^{0} / g_{1}^{0}\right) R_{\mathrm{E}}^{4}\left(r_{1} / F_{n_{\max }}\right) P_{5}^{1}(0) r^{n_{\max }-5}- \\
& -\left[\left(n_{\max }+1\right) / 2 n_{\max }\right]^{1 / 2}\left(g_{n_{\max }}^{0} / g_{1}^{0}\right) R_{\mathrm{E}}^{n_{\max }-1} \\
& \times\left(r_{1} / F_{n_{\max }}\right) P_{n_{\max }}^{1}(0)=0,
\end{aligned}
$$

where

$$
\begin{aligned}
F_{n_{\max }}= & +\sum_{n=2}^{n_{\max }}[(n+1) / 2 n]^{1 / 2}\left(g_{n}^{0} / g_{1}^{0}\right) \\
& \times P_{n}^{1}\left(\cos \theta_{0}\right) / \sin \theta_{0} .
\end{aligned}
$$

In this last equation, $F_{n_{\max }}$ denotes the parameter $F\left(\cos \theta_{0}\right)$ in the case for which the infinite summation in Eq. 9 is truncated at $n=n_{\max }$; this parameter reduces to the form given in Eq. 13 if $n_{\max }=4$. Equation 11 implies that the constant term in the polynomial Eq. 26 is non-zero if $n_{\max }$ is odd and zero if $n_{\max }$ is even. Therefore, the maximum degree of the polynomial equation in $r$ is $n_{\max }$ if $n_{\max }$ is odd and is $n_{\max }-1$ if $n_{\max }$ is even.

If specific numerical values for $g_{n}^{0}(1 \leq n \leq$ $\left.n_{\max }\right), R_{\mathrm{E}}, r_{1}$ and $\theta_{0}\left(R_{\mathrm{E}}=r_{1} \sin ^{2} \theta_{0}\right)$ are substituted into Eq. 26, the resulting polynomial can be solved numerically to determine the geocentric distance at which an individual magnetic field line crosses the equatorial plane in the general axisymmetric case $1 \leq n \leq n_{\max }$. In this paper the MATLAB polynomial function "roots" (MATLAB Reference Guide, 1992) has been used to find the roots of the polynomial Eq. 26. For a particular value of $\theta_{0}$ (or $\left.r_{1}\right)$, this MATLAB function can be used to find the real roots of the polynomial Eq. 26 for the mean (or central) values of the axisymmetric spherical harmonic coefficients $g_{n}^{0}(1 \leq n$ $\left.\leq n_{\max }\right)$ and for all $2^{n_{\max }}$ permutations of the end-points of the intervals $\left[g_{n}^{0}-\delta g_{n}^{0}, g_{n}^{0}+\delta g_{n}^{0}\right]$, as outlined in Sect. 2.4. By analogy with the special axisymmetric case $n_{\max }=4$, this procedure provides $2^{n_{\max }}$ values of $\delta r$ from which the largest value, $\delta r_{\max }$, can be determined.

\subsection{Stepwise numerical integration along magnetic field lines}

It is also possible to calculate the uncertainties in magnetic-field-line tracing in the magnetosphere by using a stepwise numerical integration procedure to trace along magnetic field lines. This approach is equivalent to obtaining a numerical solution of the differential equations that define the magnetic field lines in the magnetosphere. By analogy with the scheme 
outlined in Sect. 4.3, stepwise numerical integrations are performed for the mean (or central) values of the axisymmetric spherical harmonic coefficients $g_{n}^{0}(1 \leq n$ $\left.\leq n_{\max }\right)$ and for all $2^{n_{\max }}$ permutations of the end-points of the intervals $\left[g_{n}^{0}-\delta g_{n}^{0}, g_{n}^{0}+\delta g_{n}^{0}\right]$. This procedure provides $2_{\max }^{n}$ values of $\delta r$ from which the largest value, $\delta r_{\max }$, can be determined.

The computer program used to perform stepwise numerical integration along magnetic field lines is discussed in Paper II. It suffices to note in this section that stepwise numerical integration merely provides an alternative method of determining $\delta r_{\max }$ in the case of the axisymmetric part $(m=0)$ of the geomagnetic field of internal origin. However, no analytic equation exists for the magnetic field lines in the non-axisymmetric case $(m \neq 0)$ of the complete geomagnetic field of internal origin. In this latter case, $\delta r_{\max }$ must be determined by stepwise numerical integration along magnetic field lines. The reason for introducing stepwise numerical integration here is to show that, in the axisymmetric case $(m=0)$, this procedure provides results which are in excellent agreement with those obtained using the analytic equation derived by Backus (1988). This comparison indicates that the computer program employed to perform stepwise numerical integrations along magnetic field lines is accurate and can be used with confidence in Paper II to determine $\delta r_{\max }$ in the case of the complete geomagnetic field of internal origin.

\section{Comparison of numerical results derived by the various methods}

The various methods of determining the uncertainties in magnetic-field-line tracing in the magnetosphere, which are discussed in Sects. 4.1-4.4, are compared in this section. All numerical results presented in this paper relate solely to the axisymmetric part $(m=0)$ of the geomagnetic field of internal origin: the results for the complete non-axisymmetric $(m \neq 0)$ geomagnetic field of internal origin are presented in Paper II. Results are given for two illustrative cases in which the magnetic field line crosses the geomagnetic equator $(\theta=\pi / 2)$ at a nominal dipolar distance of either 2 or $6 R_{\mathrm{E}}$; that is $r_{1}=2 R_{\mathrm{E}}$ or $r_{1}=6 R_{\mathrm{E}}$. The results for the case $r_{1}=2 R_{\mathrm{E}}$ are likely to be reasonably realistic even when the external contributions to the geomagnetic field in the magnetosphere are included, because this case refers to the inner magnetosphere where the external contributions are relatively unimportant. The results for the case $r_{1}=6 R_{\mathrm{E}}$ are likely to be rather less realistic because the magnetic field line passes through a region of the magnetosphere in which the external contributions to the geomagnetic field have a strong influence on the magnetic-field configuration. However, it must be emphasized again that the purpose of the present pair of papers is to initiate a systematic study of the possible uncertainties in field-line tracing in the magnetosphere, starting with the geomagnetic field of internal origin (i.e. DGRF or IGRF).
Table 2. Values of $g_{n}^{0}, \delta g_{n}^{0}$ and $\left|\delta g_{n}^{0} / g_{n}^{0}\right|$ for the geomagnetic field model GSFC 1990D (after Langel et al., 1992)

\begin{tabular}{lrrl}
\hline$n$ & \multicolumn{1}{l}{$\begin{array}{l}g_{n}^{0} \\
(\mathrm{nT})\end{array}$} & \multicolumn{1}{l}{$\begin{array}{l}\delta g_{n}^{0} \\
(\mathrm{nT})\end{array}$} & $\left|\delta g_{n}^{0} / g_{n}^{0}\right|$ \\
\hline 1 & -29771.0 & 10.06 & $3.38 \times 10^{-4}$ \\
2 & -2137.0 & 8.62 & $4.03 \times 10^{-3}$ \\
3 & 1313.5 & 8.21 & $6.25 \times 10^{-3}$ \\
4 & 939.1 & 6.70 & $7.13 \times 10^{-3}$ \\
5 & -211.0 & 4.28 & $2.03 \times 10^{-2}$ \\
6 & 60.8 & 3.45 & $5.67 \times 10^{-2}$ \\
7 & 74.1 & 2.11 & $2.85 \times 10^{-2}$ \\
8 & 22.3 & 1.84 & $8.25 \times 10^{-2}$ \\
9 & 4.4 & 1.49 & $3.39 \times 10^{-1}$ \\
10 & -3.4 & 1.25 & $3.68 \times 10^{-1}$ \\
\hline
\end{tabular}

All numerical estimates of uncertainties in field-line tracing in the magnetosphere $\left(\delta r_{\max }\right)$ presented in this paper (and in Paper II) are based on the NASA Goddard Space Flight Center Model designated GSFC 1990D (Langel et al., 1992; cf. their Table 6). In all illustrative numerical calculations, each $\delta g_{n}^{0}$ is assumed to be exactly equal to the corresponding standard error in the geomagnetic field model GSFC 1990D, as outlined in Sect. 2.3. However, if each $\delta g_{n}^{0}$ is taken to be the same multiple (say $\lambda$ ) of the standard error, then the associated numerical uncertainties can be obtained approximately by multiplying the values of $\delta r_{\max }$ tabulated in this paper (and in Paper II) by the factor $\lambda$. The validity of this approximation in the general axisymmetric case can be understood in terms of the functional form of Eqs. 21-25, provided that the inequalities $\left(g_{n}^{0} / g_{1}^{0}\right) \ll 1$ and $\left(\delta g_{n}^{0} / g_{n}^{0}\right) \ll 1$ are satisfied for at least the important spherical harmonic coefficients. For completeness, the values of $g_{n}^{0}, \delta g_{n}^{0}$ and $\left|\delta g_{n}^{0} / g_{n}^{0}\right|$ for the geomagnetic field model GSFC 1990D are presented in Table 2 . It is clear from this table that third-order terms in $\delta g_{n}^{0} / g_{n}^{0}\left(1 \leq n \leq n_{\max }\right)$ can be neglected, which justifies the approximations made in Sect. 4.2 and Appendix A.

Tables 3 and 4 present numerical estimates of the maximum uncertainty $\delta r_{\max }$ in the geocentric distance at which a magnetic field line crosses the geomagnetic equator $(\theta=\pi / 2)$ for the axisymmetric part $(m=0)$ of the geomagnetic field of internal origin. Table 3 provides results for the special axisymmetric case $n_{\max }=4$, which is discussed in detail in Sects. 3.2 and 4.1. Similarly, Table 4 provides results for the general axisymmetric case with $n_{\max }=10$, which is discussed in detail in Sect. 4.3. Both tables present estimates of $\delta r_{\max }$ for nominal

Table 3. Comparison between various numerical estimates for $\delta r_{\max }$ at the geomagnetic equator in the special axisymmetric case $n_{\max }=4$

\begin{tabular}{lcl}
\hline Method of solution & $\begin{array}{l}\delta r_{\max }\left(r_{1}=2 R_{\mathrm{E}}\right) \\
(\mathrm{km})\end{array}$ & $\begin{array}{l}\delta r_{\max }\left(r_{1}=6 R_{\mathrm{E}}\right) \\
(\mathrm{km})\end{array}$ \\
\hline Exact (Mathematica) & 7.2183 & 48.3942 \\
Polynomial (MATLAB) & 7.2183 & 48.3942 \\
Tracing Program & 7.2184 & 48.3945 \\
Approx. Eqns & 7.2760 & 48.4597 \\
\hline
\end{tabular}


Table 4. Comparison between various numerical estimates for $\delta r_{\max }$ at the geomagnetic equator in the general axisymmetric case $n_{\max }=10$

\begin{tabular}{|c|c|c|}
\hline Method of solution & $\begin{array}{l}\delta r_{\max } \\
(\mathrm{km})\end{array} \quad\left(r_{1}=2 R_{\mathrm{E}}\right)$ & $\begin{array}{l}\delta r_{\max } \\
(\mathrm{km})\end{array}\left(r_{1}=6 R_{\mathrm{E}}\right)$ \\
\hline Polynomial (MATLAB) & 9.3029 & 65.4833 \\
\hline Tracing Program & 9.3030 & 65.4839 \\
\hline Approx. Eqns & 9.3876 & 65.5684 \\
\hline
\end{tabular}

dipolar crossing distances of $r_{1}=2 R_{\mathrm{E}}$ and $r_{1}=6 R_{\mathrm{E}}$, as already noted. In these tables the following abbreviations are used to denote the methods by which the numerical estimates for $\delta r_{\max }$ are derived. Exact (Mathematica) refers to the exact algebraic solution of the cubic equation, Eq. 14, which is obtained using the computer algebra package Mathematica (Wolfram, 1988) and is defined by Eq. 16. Polynomial (MATLAB) refers to the numerical solution of the tenth-degree polynomial, Eq. 26, using the MATLAB polynomial function "roots" (MATLAB Reference Guide, 1992). Tracing Program refers to numerical results obtained using the computer program that performs stepwise numerical integration, as discussed in detail in Sect. 3 of Paper II. Approx. Eqns refers to the approximate algebraic solution of Eq. 20, defined by Eqs. 21-25.

It is clear from Tables 3 and 4 that all methods of estimating $\delta r_{\max }$ give numerical results that are in remarkably good agreement. Table 3 is included because the special axisymmetric case $n_{\max }=4$ is the highestdegree case for which the equations can be solved exactly using algebraic techniques. Thus the only possible source of numerical inaccuracy in the values of $\delta r_{\text {max }}$ presented in the first row of Table 3 arises solely from the insertion of numerical values in an algebraic expression. However, the values of $\delta r_{\max }$ based on an iterative numerical procedure for finding the roots of the cubic equation, Eq. 14, and on stepwise numerical integration along magnetic field lines (i.e. rows 2 and 3 of Table 3) only differ from the "exact" estimates (i.e. row 1 of Table 3) by less than $1 \mathrm{~m}$. Therefore, the technique of stepwise numerical integration along field lines does not result in significant cumulative numerical errors. Even the approximate algebraic solution of Eq. 20 with $n_{\max }=4$, which is only valid to second order in the small terms $\delta g_{n}^{0} / g_{n}^{0}\left(1 \leq n \leq n_{\max }\right)$, is adequately accurate for many purposes.

It can be seen from the numerical estimates for $\delta r_{\max }$ presented in Table 4 that similar conclusions hold for the general axisymmetric case $n_{\max }=10$, which corresponds to the entire axisymmetric part of the geomagnetic field model GSFC 1990D (Langel et al., 1992). The differences between corresponding numbers in Tables 3 and 4 represent the additional contributions to $\delta r_{\max }$ that arise from higher-degree axisymmetric multipoles in the range $5 \leq n \leq 10$. The fact that the estimates of $\delta r_{\max }$ obtained using the tracing program in the case $n_{\max }=10$ are in remarkably good agreement with those obtained quite independently from the solution of the analytic equation derived by Backus (1988) implies that the tracing program is very accurate. This conclusion is of vital importance in Paper II, which considers the uncertainties in magnetic-field-line tracing in the magnetosphere for the complete geomagnetic field of internal origin. In this non-axisymmetric case, no analytic equation exists for the magnetic field lines, and uncertainties in field-line tracing can only be estimated using stepwise numerical integration along magnetic field lines.

The numerical estimates for $\delta r_{\max }$ presented in both Tables 3 and 4 imply that classical pertubation theory (i.e. Approx. Eqns) yields estimates which are significantly less accurate than those calculated by any of the numerical methods. However, classical pertubation theory has the great compensating advantage that Eqs. 21-25 give an indication of how $\delta r$ varies as a function of the (axisymmetric) spherical harmonic coefficients $\left(g_{n}^{0}\right)$ and their associated errors $\left(\delta g_{n}^{0}\right)$, at least for $\left|g_{n}^{0} / g_{1}^{0}\right| \ll 1\left(2 \leq n \leq n_{\max }\right)$ and $\left|\delta g_{n}^{0} / g_{n}^{0}\right| \ll 1 \quad(1 \leq n$ $\leq n_{\max }$.

\section{Interval mapping for the axisymmetric part of the internal geomagnetic field}

It is assumed implicitly in Sects. 2.4 and 4.1-4.4 that in the axisymmetric case $(m=0) \delta r_{\max }$ can be determined simply by considering every possible permutation of the "lower" and "upper" end-points, $g_{n}^{0}-\delta g_{n}^{0}, g_{n}^{0}+\delta g_{n}^{0}$, of the intervals of real numbers $\left[g_{n}^{0}-\delta g_{n}^{0}, g_{n}^{0}+\delta g_{n}^{0}\right]$ for $1 \leq n \leq n_{\max }$. Stated alternatively, it is assumed that the maximum value of $\delta r\left(\delta r_{\max }\right)$ in the set of values $\{r+\delta r\}$ corresponds to a particular permutation of the endpoints $\left(g_{n}^{0} \pm \delta g_{n}^{0}\right)$ in the coefficient intervals $\left[g_{n}^{0}+\Delta g_{n}^{0}\right]$, where $\delta r \in \mathbb{R}$ and $-\delta g_{n}^{0} \leq \Delta g_{n}^{0} \leq+\delta g_{n}^{0}$. This implicit assumption effectively reduces a continuous interval mapping problem to a discrete one, which enables calculations of the uncertainties in magneticfield-line tracing in the magnetosphere to be undertaken with finite computing resources. Since it is intuitively clear that the axisymmetric part of the spherical harmonic expansion of the internal geomagnetic field is a well-behaved function of the spherical harmonic coefficients, this implicit assumption seems reasonable physically. An argument is developed in the following section to demonstrate numerically that interior points in the coefficient intervals apparently do not map to exterior points in the uncertainty interval for $r$.

\subsection{Conjecture 1: interval mapping in the axisymmetric case $(\mathrm{m}=0)$}

Consider the following conjecture for the axisymmetric part of the internal geomagnetic field $(m=0)$, which is designated Conjecture 1. (Conjecture 2 is presented in Paper II.) Conjecture 1: For the contemporary geomagnetic field, there do not exist points $g_{n}^{0}+\Delta g_{n}^{0}$, where $-\delta g_{n}^{0} \leq \Delta g_{n}^{0} \leq+\delta g_{n}^{0}\left(\delta g_{n}^{0} \geq 0\right)$, in the interval $\left[g_{n}^{0}-\delta g_{n}^{0}, g_{n}^{0}+\delta g_{n}^{0}\right]^{n}$ for which $\mathscr{P}\left(g_{n}^{0}+\Delta g_{n}^{0}\right)=r+\Delta r$ such that $|\Delta r|>\left|\delta r_{\max }\right|$. The symbol $\mathscr{P}$ is used to denote the "transformation" from coefficient intervals 
to uncertainty interval using the polynomial Eq. 26 to find $\Delta r$ for given $\Delta g_{n}^{0}\left(1 \leq n \leq n_{\max }\right)$, as discussed in Sect. 4.3. Note that with this definition, $\mathscr{P}\left(g_{n}^{0}\right)=r$ and $\mathscr{P}\left(g_{n}^{0} \pm \delta g_{n}^{0}\right)=r+\delta r_{\max }$.

The credibility of Conjecture 1 is established by calculating $\delta r$ by two different methods and comparing the results as follows. In Method A it is supposed that the interval of real numbers $\left[g_{n}^{0}-\delta g_{n}^{0}, g_{n}^{0}+\delta g_{n}^{0}\right]$ is represented by the five sample points (real numbers) $g_{n}^{0}$, $g_{n}^{0} \pm(1 / 2) \delta g_{n}^{0}$ and $g_{n}^{0} \pm \delta g_{n}^{0}\left(\delta g_{n}^{0} \geq 0\right)$, rather than by just the two end-points $g_{n}^{0} \pm \delta \bar{g}_{n}^{0}$. By an obvious extension of the arguments presented in Sect. 2.4, there are $5^{n_{\max }}$ "limiting geomagnetic-field models" in this case, since $N=n_{\max }$ if $m=0$. The use of the term "limiting geomagnetic-field model" for the three interior sample points $g_{n}^{0}$ and $g_{n}^{0} \pm(1 / 2) \delta g_{n}^{0}$ tacitly acknowledges the possibility that Conjecture 1 might be incorrect. The MATLAB polynomial function "roots" (MATLAB Reference Guide, 1992) is then used to solve the corresponding $5^{10}$ polynomials defined by Eqs. 26 and 27 in the general axisymmetric case $n_{\max }=10$, using the axisymmetric part of the internal geomagnetic-field model GSFC 1990D (cf. Table 2). This procedure generates the $5^{10}$ values of $\delta r^{A}$ that correspond to selecting all $5^{10}$ permutations of the five sample points $g_{n}^{0}$, $g_{n}^{0} \pm(1 / 2) \delta g_{n}^{0}, g_{n}^{0} \pm \delta g_{n}^{0}$, according to Method A. Another MATLAB routine is used to arrange these $5^{10}$ values of $\delta r^{A}$ as a monotonic increasing sequence $\left\{\delta r_{\text {min }}^{A}\right.$, $\left.\ldots, \delta r_{\max }^{A}\right\}$, where $\delta r_{\min }^{A}$ and $\delta r_{\max }^{A}$ denote the smallest and largest values of $\delta r^{A}$ calculated by Method A.

In Method B the MATLAB polynomial function "roots" is used to solve the $5^{10}$ polynomials generated by Eqs. 26 and 27 if each of the ten coefficients $g_{n}^{0}(1 \leq n \leq 10)$ in the GSFC $1990 \mathrm{D}$ model is assumed to have a random error $\Delta g_{n}^{0}$ in the error interval $\left[-\delta g_{n}^{0}\right.$, $\left.+\delta g_{n}^{0}\right]$ and this random selection of the ten errors $\Delta g_{n}^{0}$ is repeated $5^{10}$ times. The random errors $\Delta g_{n}^{0}$ are generated using the NAG routine G05DAF (NAG Fortran Library Manual, 1988), which produces sequences of pseudo-random numbers taken from a uniform distribution in the interval $\left[g_{n}^{0}-\delta g_{n}^{0}\right.$, $\left.g_{n}^{0}+\delta g_{n}^{0}\right]$. The resulting $5^{10}$ values of $\delta r^{B}$ are then arranged as a monotonic increasing sequence $\left\{\delta r_{\min }^{B}\right.$, $\left.\delta r_{\max }^{B}\right\}$, where $\delta r_{\min }^{B}$ and $\delta r_{\max }^{B}$ denote the smallest and largest values of $\delta r^{B}$ calculated by Method B.

Lastly, the values of $\delta r_{\max }^{A}$ and $\delta r_{\max }^{B}$ are compared with each other and with the value of $\delta r_{\max }$ obtained by considering all $2^{10}$ possible permutations of the endpoints of the intervals $\left[g_{n}^{0}-\delta g_{n}^{0}, g_{n}^{0}+\delta g_{n}^{0}\right]$ for $1 \leq n \leq 10$, as described in Sect. 2.4; similarly for

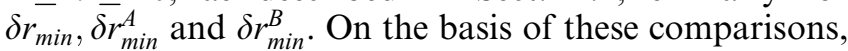
Conjecture 1 is accepted (at least as a working hypothesis) if the four conditions $\delta r_{\max }^{A}=\delta r_{\max }$, $\delta r_{\text {max }}^{A}>\delta r_{\text {max }}^{B}, \delta r_{\text {min }}^{A}=\delta r_{\text {min }}$ and $\delta r_{\text {min }}^{A}<\delta r_{\text {min }}^{B}$ hold. These four conditions have been satisfied in every case examined in this study. Therefore, a set of ten internal points, lying in the axisymmetric coefficient intervals $\left[g_{n}^{0}-\delta g_{n}^{0}, g_{n}^{0}+\delta g_{n}^{0}\right]$ for $1 \leq n \leq 10$, apparently always maps to a value of $\delta r$ lying in the uncertainty interval $\left[\delta r_{\min }, \delta r_{\max }\right]$.
The argument presented here certainly does not constitute a completely rigorous mathematical proof of Conjecture 1. In fact, the credibility of Conjecture 1 has only been investigated numerically for the spherical harmonic coefficients $\left(g_{n}^{0}\right)$ and associated errors $\left(\delta g_{n}^{0}\right)$ that specify the axisymmetric part of the GSFC 1990D geomagnetic reference field (Langel et al., 1992). Nevertheless, Conjecture 1 is equally credible for the axisymmetric part of any contemporary geomagnetic reference field. This conclusion follows from the fact that the functional form of Eq. 21 , together with the definitions in Eqs 22-25, corroborates the credibility of Conjecture 1 under the conditions $\left|g_{n}^{0} / g_{1}^{0}\right| \ll 1\left(2 \leq n \leq n_{\max }\right)$ and $\left|\delta g_{n}^{0} / g_{n}^{0}\right| \ll 1 \quad\left(1 \leq n \leq n_{\max }\right)$, which hold for all contemporary geomagnetic reference fields. Finally, however, it must be stressed that no conclusion is being propounded in this paper about the credibility of Conjecture 1 for purely arbitrary values of the axisymmetric spherical harmonic coefficients $\left(g_{n}^{0}\right)$ and their associated errors $\left(\delta g_{n}^{0}\right)$. Further research is required to elucidate the general case.

\subsection{Graphical presentation of results for the general axisymmetric case}

The theoretical methods described in Sect. 6.1 are illustrated in this section by presenting histograms of the monotonic increasing sequences $\left\{\delta r^{A}\right\}$ and $\left\{\delta r^{B}\right\}$, obtained by solving the $5^{10}$ polynomials defined by Eqs. 26 and 27 according to Methods A and B. Histograms are presented for the same two illustrative cases as considered in Sect. 5, namely those for which the geomagnetic field line crosses the geomagnetic equator $(\theta=\pi / 2)$ at a nominal dipolar distance of either 2 or $6 R_{\mathrm{E}}$. Figures 1 and 2 present histograms for the cases $r_{1}=2 R_{\mathrm{E}}$ and $r_{1}=6 R_{\mathrm{E}}$, respectively. Note that $\delta r$ can be either positive or negative, as indicated in Sect. 4.2. In each figure the continuous curve represents a normal (or Gaussian) distribution with the same mean and variance as the corresponding histogram. For the case $r_{1}=2 R_{\mathrm{E}}$, Fig. 1a presents the histogram of the monotonic increasing sequence $\left\{\delta r_{i}^{A}\right\}$ in which the elements $\delta r_{i}^{A}\left(1 \leq i \leq 5^{10}\right)$ are determined by solving the $5^{10}$ tenth-degree polynomials associated with all possible permutations of the sample coefficients $g_{n}^{0}, g_{n}^{0} \pm$ $(1 / 2) \delta g_{n}^{0}, g_{n}^{0} \pm \delta g_{n}^{0}(1 \leq n \leq 10)$, as outlined in Method A. Similarly, Fig. $1 \mathrm{~b}$ presents the histogram of the monotonic increasing sequence $\left\{\delta r_{i}^{B}\right\}$ in which the elements $\delta r_{i}^{B}\left(1 \leq i \leq 5^{10}\right)$ are determined by solving the $5^{10}$ tenth-degree polynomials arising from $5^{10}$ selections of the ten random errors $\Delta g_{n}^{0}$ in $g_{n}^{0}$ that are located randomly in the ten corresponding error intervals $\left[-\delta g_{n}^{0},+\delta g_{n}^{0}\right]$, as outlined in Method B. The histograms presented in Fig. 2a,b are calculated in exactly the same way but with $r_{1}=6 R_{\mathrm{E}}$.

Figures $1 \mathrm{a}$ and $2 \mathrm{a}$ give the actual numerical values of $\delta r_{\max }^{A}$ and $\delta r_{\min }^{A}$ as well as the mean and standard deviation (std) of the histogram derived by Method A. Similarly, Figs. $1 b$ and $2 b$ give the actual numerical 

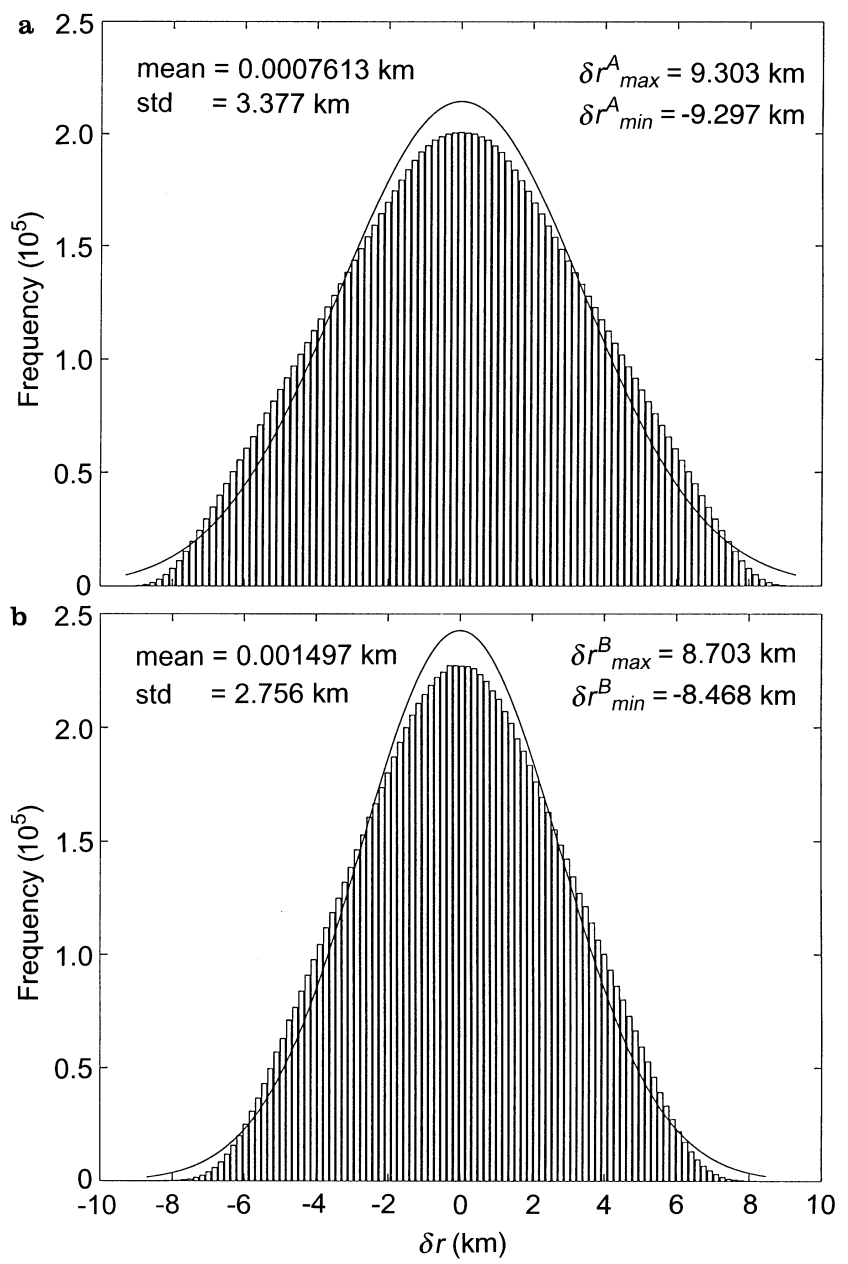

Fig. 1a,b. Histograms of the uncertainties $(\delta r)$ in the geocentric distance at which a magnetic field line crosses the geomagnetic (geographic) equator in the general axisymmetric case $n_{\max }=10$ for $r_{1}=2 R_{\mathrm{E}}$. a $\delta r^{A}$ calculated by Method A, which involves solving the $5^{10}$ tenth-degree polynomials associated with all permutations of the sample coefficients $g_{n}^{0}, g_{n}^{0} \pm(1 / 2) \delta g_{n}^{0}, g_{n}^{0} \pm \delta g_{n}^{0} \quad(1 \leq n \leq 10)$. b $\delta r^{B}$ calculated by Method $\mathrm{B}$, which involves solving the $5^{10}$ tenthdegree polynomials arising from $5^{10}$ selections of ten (random) errors $\Delta g_{n}^{0}$ in $g_{n}^{0}(1 \leq n \leq 10)$, located randomly in the ten corresponding error intervals $\left[-\delta g_{n}^{0},+\delta g_{n}^{0}\right]$

values of $\delta r_{\max }^{B}$ and $\delta r_{\min }^{B}$ as well as the mean and standard deviation of the histogram derived by Method B. The means in Figs. 1 and 2 differ from zero because the geomagnetic field decreases non-linearly with geocentric distance. Moreover, since the geomagnetic field decreases less rapidly with increasing geocentric distance, $\delta r_{\max }>\left|\delta r_{\min }\right|$ and the statistical mean is always greater than zero. It is clear, both by visual inspection of the histograms in Figs. 1 and 2 and by comparison of the actual numerical values, that $\delta r_{\max }^{A}>\delta r_{\max }^{B}$ and $\delta r_{\text {min }}^{A}<\delta r_{\text {min }}^{B}$. Moreover, $\delta r_{\text {max }}^{A}={ }^{\max } \delta r_{\max }$ and $\delta r_{\min }^{A}=\delta r_{\min }$, where $\delta r_{\max }$ and $\delta r_{\min }$ denote the maximum and minimum values of $\delta r$ derived by repeating Method A for the situation in which each interval of real numbers $\left[g_{n}^{0}-\delta g_{n}^{0}, g_{n}^{0}+\delta g_{n}^{0}\right]$ is represented by just the two (sample) end-points $g_{n}^{0} \pm \delta g_{n}^{0}$, rather than by all five sample points $g_{n}^{0}, g_{n}^{0} \pm(1 / 2) \delta g_{n}^{0}, g_{n}^{0} \pm \delta g_{n}^{0}(1 \leq n \leq 10)$.
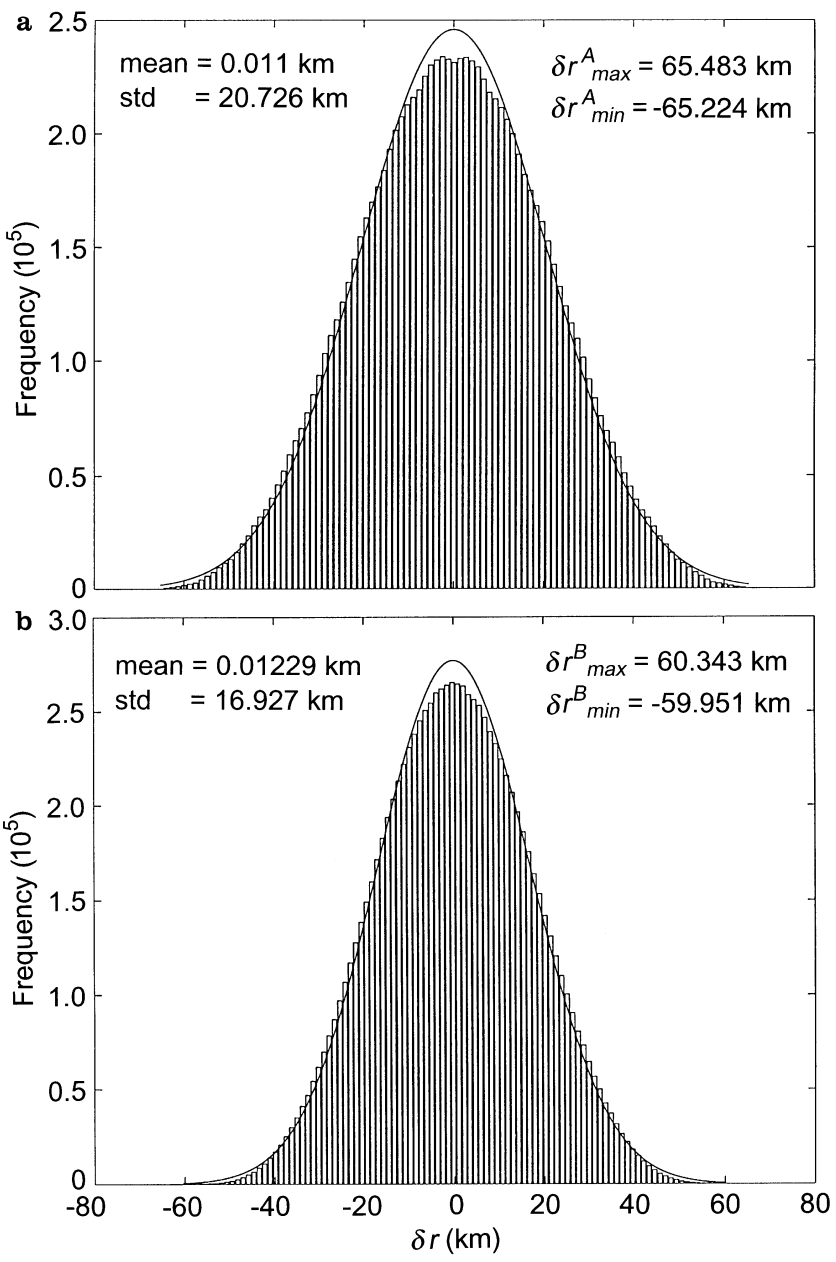

Fig. 2a,b. Same as Fig. 1, but for $r_{1}=6 R_{\mathrm{E}}$

The veracity of this last statement can be confirmed by comparing the numerical values of $\delta r_{\max }^{A}$ presented in the top right-hand corners of Figs. 1a and $2 \mathrm{a}$ with the corresponding numerical values of $\delta r_{\max }$ presented in Table 4. Since $\delta r_{\max }$ and $\delta r_{\min }$ are always associated with particular permutations of the end-points of the coefficient intervals $\left[g_{n}^{0}-\delta g_{n}^{0}, g_{n}^{0}+\delta g_{n}^{0}\right]$, internal points in these coefficient intervals do not yield values of $\delta r$ that lie outside the uncertainty interval $\left[\delta r_{\min }, \delta r_{\max }\right]$. Therefore, the credibility of Conjecture 1 is established.

\section{Summary and conclusions}

The goal of this investigation is to begin a systematic study of the uncertainties in magnetic-field-line tracing in the magnetosphere that arise from uncertainties in the specification of the six main sources of magnetic field in the magnetosphere, namely: (1) currents flowing in the Earth's liquid metallic outer core; (2) ionospheric currents; (3) field-aligned (or Birkeland) currents; (4) ring currents; (5) magnetopause currents; and (6) magnetotail currents (Stern and Tsyganenko, 1992). It 
seems sensible to commence such a systematic study with a detailed examination of the uncertainties in fieldline tracing in the magnetosphere produced solely by possible errors in the specification of the geomagnetic field of internal origin (Sect. 2). The primary purpose of this initial investigation is to estimate these uncertainties by using one of the few published models of the geomagnetic field that presents both the spherical harmonic coefficients and their standard errors (Langel et al., 1989, 1992). Because of the considerable complexity in computing these uncertainties in field-line tracing for the complete geomagnetic field of internal origin, attention is focused in this preliminary paper on the uncertainties that result from the standard errors in just the axisymmetric part of the internal geomagnetic field (Sect. 3).

An exact analytic equation exists for the magnetic field lines of an arbitrary linear combination of axisymmetric multipoles (Sect. 3.1). This equation is used to derive accurate numerical estimates of the uncertainties in magnetic-field-line tracing that are due to the standard errors in the axisymmetric spherical harmonic coefficients (i.e. $g_{n}^{0} \pm \delta g_{n}^{0}$ ) published by Langel et al. (1992). In the special axisymmetric case arising from the addition of just the first four axisymmetric multipoles $\left(n_{\max }=4\right.$ and hence $\left.1 \leq n \leq 4\right)$, namely a dipole $\left(g_{1}^{0}\right)$, a quadrupole $\left(g_{2}^{0}\right)$, an octupole $\left(g_{3}^{0}\right)$ and a sedecimupole $\left(g_{4}^{0}\right)$, the resulting quartic equation can be solved algebraically (Sect. 3.2). However, the roots of this quartic equation are extremely complicated and therefore the discussion is restricted to the solution of the simpler cubic equation for the geocentric distance at which an individual magnetic field line crosses the geomagnetic equatorial plane (Sect. $4.1)$. In the general axisymmetric case $\left(n_{\max }=10\right.$ and hence $1 \leq n \leq 10$ ), the uncertainties in the geocentric distance at which a field line crosses the geomagnetic equator are determined numerically by solving the corresponding tenth-degree polynomial equation (Sect. 4.3). Even in the general axisymmetric case, an approximate algebraic solution can be obtained if it is assumed that $\left|g_{n}^{0} / g_{1}^{0}\right| \ll 1$ for $2 \leq n \leq 10$ and $\left|\delta g_{n}^{0} / g_{1}^{0}\right| \ll 1$ for $1 \leq n \leq 10$ (Sect. 4.2). Finally, it is also possible to calculate the uncertainties in magneticfield-line tracing in the magnetosphere by using a stepwise numerical integration procedure to trace along magnetic field lines (Sect. 4.4).

The various methods of determining the uncertainties in magnetic-field-line tracing for the axisymmetric part $(m=0)$ of the internal geomagnetic field are compared quantitatively (Sect. 5). Numerical results are presented for two illustrative cases in which the magnetic field line crosses the geomagnetic equator at a nominal dipolar distance of either 2 or $6 R_{\mathrm{E}}$ (Tables 3 and 4 ). All these numerical estimates of the maximum uncertainty $\left(\delta r_{\max }\right)$ in field-line tracing in the magnetosphere are based on the NASA GSFC 1990D model (Langel et al., 1992) of the internal geomagnetic field (Sect. 2.3 and Table 2). It is clear from the actual numbers in Tables 3 and 4 that the various methods of estimating the uncertainties $\delta r_{\max }$ give results that are in excellent agreement. For just the axisymmetric part of the internal geomagnetic field $\left(n_{\max }=10\right)$, the maximum characteristic uncertainty in the geocentric distance of a field line that crosses the magnetic equator at a nominal dipolar distance of $2 R_{\mathrm{E}}$ is typically $\pm 10 \mathrm{~km}$ (Table 4 ). The corresponding characteristic uncertainty for a field line that crosses the equator at a nominal dipolar distance of $6 R_{\mathrm{E}}$ is typically $\pm 70 \mathrm{~km}$ (Table 4 ).

Numerical estimates of the uncertainties in magneticfield-line tracing in the magnetosphere, which are calculated in this paper for just the axisymmetric part of the internal geomagnetic field, should be regarded as "first approximations", in the sense that such estimates are only as accurate as the published standard errors in the set of axisymmetric spherical harmonic coefficients (Langel et al., 1989, 1992). However, all the procedures developed in this preliminary paper can be applied to the derivation of more realistic estimates of the uncertainties in magnetic-field-line tracing in the magnetosphere, following further progress in the determination of more accurate standard errors in the spherical harmonic coefficients.

Finally, it should be emphasized that the values of $\delta r_{\max }$ derived by stepwise numerical integration along magnetic field lines are essentially identical to those derived independently by the iterative numerical procedure for finding the roots of the polynomial equation due to Backus (1988). The excellent agreement between results derived by these two different techniques confirms that the computer program used for stepwise numerical integration along magnetic field lines is very accurate. This conclusion is of critical importance in the following paper (Paper II), which considers the uncertainties in magnetic-field-line tracing in the magnetosphere for the complete geomagnetic field of internal origin. In the general non-axisymmetric case $(m \neq 0)$, no analytic equation exists for the magnetic field lines and uncertainties in field-line tracing can only be estimated by using stepwise numerical integration along magnetic field lines, as discussed in detail in Paper II.

Acknowledgements. The authors are greatly indebted to Dr D. R. Barraclough and Dr M. A. Hapgood for valuable advice and thank Mr S. R. Crothers, Mr V.N. Davda and Mrs J. Foster for considerable help with the preparation of the paper. The research reported in this paper was undertaken while JC and JRS pursued consecutive twelve-month periods of Professional Training at the Rutherford Appleton Laboratory as part of the BSc. Honours Degree Course in Mathematics at Coventry University; JRS continued this research as a fourth-year project.

Topical Editor K.-H. Glaßmeier thanks D. R. Barraclough, G. Backus and another referee for their help in evaluating this paper.

\section{Appendix A. Approximate solution of Eq. 20}

It is shown in Sect. 4.2 that, in the general axisymmetric case $2 \leq n \leq n_{\max }$, the geocentric distance at which an individual magnetic field line crosses the equatorial plane $(\theta=\pi / 2)$ is defined by Eq. 20, namely 


$$
r=r_{1} \frac{\llbracket 1+\sum_{n=2}^{n_{\max }}[(n+1) / 2 n]^{1 / 2}\left(g_{n}^{0} / g_{1}^{0}\right)\left(R_{\mathrm{E}} / r\right)^{n-1} P_{n}^{1}(0) \rrbracket}{\llbracket 1+\sum_{n=2}^{n_{\max }}[(n+1) / 2 n]^{1 / 2}\left(g_{n}^{0} / g_{1}^{0}\right) P_{n}{ }^{1}\left(\cos \theta_{0}\right) / \sin \theta_{0} \rrbracket} .
$$

Assume that $\left|g_{n}^{0} / g_{1}^{0}\right| \ll 1$ for $2 \leq n \leq n_{\max }$, which is certainly valid for the contemporary geomagnetic field of internal origin because the dipole term $\left(g_{1}^{0}\right)$ predominates. Then, to a first approximation, the solution of Eq. Al is $r \approx r_{1}$, which is otherwise clear from Eq. 5 with $\theta=\pi / 2$.

If $r$ is replaced by $r_{1}$ on the right-hand side of Eq. A1, the second approximation for $r$ is given by

$r \approx r_{1}\left[1+\sum_{n=2}^{n_{\max }} a_{n} G_{n}\right]\left[1+\sum_{n=2}^{n_{\max }} b_{n} G_{n}\right]^{-1}$

where, for $n \geq 2$,

$G_{n}=g_{n}^{0} / g_{1}^{0}$

$a_{n}=[(n+1) / 2 n]^{1 / 2}\left(R_{\mathrm{E}} / r_{1}\right)^{n-1} P_{n}^{1}(0)$

and

$b_{n}=[(n+1) / 2 n]^{1 / 2} P_{n}^{1}\left(\cos \theta_{0}\right) / \sin \theta_{0}$.

Equation A2 can be written in the form

$r \approx r_{1} \llbracket 1+\left[\sum_{n=2}^{n_{\max }}\left(a_{n}-b_{n}\right) G_{n}\right]\left[1+\sum_{n=2}^{n_{\max }} b_{n} G_{n}\right]^{-1} \rrbracket$.

On the assumption that $\left|\sum_{n=2}^{n_{\max }} b_{n} G_{n}\right|<1$, the binomial theorem can be used to expand the second term in square brackets on the right-hand side of Eq. A6; this gives

$$
\begin{aligned}
r \approx r_{1} \llbracket & +\left[\sum_{n=2}^{n_{\max }}\left(a_{n}-b_{n}\right) G_{n}\right]\left[\sum_{N^{*}=1}^{\infty}(-1)^{N^{*}-1}\right. \\
& \left.\times\left\{\sum_{n=2}^{n_{\max }} b_{n} G_{n}\right\}^{N^{*}-1}\right] \rrbracket
\end{aligned}
$$

where $N^{*} \in \mathbb{I}^{+}$.

It is now necessary to allow for the uncertainties ("error bars") $\delta g_{n}^{0}$ in the axisymmetric spherical harmonic coefficients $g_{n}^{0}\left(1 \leq n \leq n_{\max }\right)$. Contrary to the convention adopted in Sect. 2.3, it is assumed here that each $\delta g_{n}^{0}$ can be positive or negative; this assumption eliminates the need to use \pm signs throughout the analysis. If $\delta r$ denotes the uncertainty in the geocentric distance at which an individual magnetic field line crosses the equatorial plane, arising from the
D. M. Willis et al.: Uncertainties in magnetospheric field-line tracing

uncertainties $\delta g_{n}^{0}\left(1 \leq n \leq n_{\max }\right)$, then Eq. A7 implies that

$$
\begin{aligned}
r+\delta r \approx r_{1} \llbracket 1 & +\left[\sum_{n=2}^{n_{\max }}\left(a_{n}-b_{n}\right) G_{n}\left(1+\delta G_{n} / G_{n}\right)\right] \\
& \times\left[\sum _ { N ^ { * } = 1 } ^ { \infty } ( - 1 ) ^ { N ^ { * } - 1 } \left\{\sum_{n=2}^{n_{\max }} b_{n} G_{n}\right.\right. \\
& \left.\left.\times\left(1+\delta G_{n} / G_{n}\right)\right\}^{N^{*}-1}\right] \rrbracket
\end{aligned}
$$

where, from Eq. A3,

$$
\begin{aligned}
G_{n}\left(1+\delta G_{n} / G_{n}\right)= & G_{n}\left(1+\delta g_{n}^{0} / g_{n}^{0}\right)\left(1+\delta g_{1}^{0} / g_{1}^{0}\right)^{-1}, \\
& (n \geq 2) .
\end{aligned}
$$

Since $\left|\delta g_{n}^{0} / g_{n}^{0}\right| \ll 1$ for $1 \leq n \leq n_{\max }$, it follows from Eq. A9 that

$$
\begin{aligned}
\delta G_{n} / G_{n}= & \left(\delta g_{n}^{0} / g_{n}^{0}-\delta g_{1}^{0} / g_{1}^{0}\right)-\left(\delta g_{1}^{0} / g_{1}^{0}\right) \\
& \times\left(\delta g_{n}^{0} / g_{n}^{0}-\delta g_{1}^{0} / g_{1}^{0}\right),
\end{aligned}
$$

to second order in the small terms $\delta g_{1}^{0} / g_{1}^{0}$ and $\delta g_{n}^{0} / g_{n}^{0}$.

If the binomial theorem is used to expand the expression $\left(\sum_{n=2}^{n_{\max }} b_{n} G_{n}+\sum_{n=2}^{n_{\max }} b_{n} \delta G_{n}\right)^{N^{*}-1}$ up to the third term, Eq. A8 can be expressed in the form

$$
\begin{aligned}
r+\delta r \approx r_{1} \llbracket 1 & +\left[\sum_{n=2}^{n_{\max }}\left(a_{n}-b_{n}\right) G_{n}\left(1+\delta G_{n} / G_{n}\right)\right] \\
& \times\left[\sum _ { N ^ { * } = 1 } ^ { \infty } ( - 1 ) ^ { N ^ { * } - 1 } \left\{\left(\sum_{n=2}^{n_{\max }} b_{n} G_{n}\right)^{N^{*}-1}\right.\right. \\
& +\left(N^{*}-1\right)\left(\sum_{n=2}^{n_{\max }} b_{n} G_{n}\right)^{N^{*}-2}\left(\sum_{n=2}^{n_{\max }} b_{n} \delta G_{n}\right) \\
& +\frac{\left(N^{*}-1\right)\left(N^{*}-2\right)}{2} \\
& \left.\left.\times\left(\sum_{n=2}^{n_{\max }} b_{n} G_{n}\right)^{N^{*}-3}\left(\sum_{n=2}^{n_{\max }} b_{n} \delta G_{n}\right)^{2}\right\}\right] \rrbracket
\end{aligned}
$$

which is accurate to second-order terms in $\delta G_{n}$, viz. $\delta G_{n_{i}} . \delta G_{n_{j}}\left(2 \leq n_{i} \leq n_{\max }\right)$ and $\left(2 \leq n_{j} \leq n_{\max }\right)$, but neglects all higher-order terms. Using Eq. A7 and neglecting the third-order term in $\delta G_{n}$, it can be shown after some algebraic manipulation that

$$
\begin{aligned}
\delta r \approx & r_{1} \sum_{N^{*}=1}^{N_{\max }^{*}}(-1)^{N^{*}-1}\left[\left[\left(N^{*}-1\right)\left(\sum_{n=2}^{n_{\max }} b_{n} G_{n}\right)^{N^{*}-3}\right.\right. \\
& \times\left(\sum_{n=2}^{n_{\max }} b_{n} \delta G_{n}\right)\left\{[ \sum _ { n = 2 } ^ { n _ { \operatorname { m a x } } } ( a _ { n } - b _ { n } ) G _ { n } ] \left[\left(\sum_{n=2}^{n_{\max }} b_{n} G_{n}\right)\right.\right. \\
& \left.+\frac{\left(N^{*}-2\right)}{2}\left(\sum_{n=2}^{n_{\max }} b_{n} \delta G_{n}\right)\right]+\left[\sum_{n=2}^{n_{\max }}\left(a_{n}-b_{n}\right) \delta G_{n}\right]
\end{aligned}
$$




$$
\begin{aligned}
& \left.\times\left(\sum_{n=2}^{n_{\max }} b_{n} G_{n}\right)\right\}+\left(\sum_{n=2}^{n_{\max }} b_{n} G_{n}\right)^{N^{*}-1} \\
& \left.\times\left[\sum_{n=2}^{n_{\max }}\left(a_{n}-b_{n}\right) \delta G_{n},\right]\right]
\end{aligned}
$$

where the summations over the infinite range $1 \leq N^{*}<\infty$ are now restricted to the finite range $1 \leq N^{*} \leq N_{\max }^{*}$, by analogy with the summations over the finite range $2 \leq n \leq n_{\max }$.

\section{References}

Akasofu, S.-I., and S. Chapman, Solar-Terrestrial Physics, Oxford University Press, London, 1972.

Albert, R. D., Nearly monoenergetic electron fluxes detected during a visible aurora, Phys. Rev. Lett., 18, 369-372, 1967.

Alfvén, H., and C.-G. Fälthammar, Cosmical Electrodynamics, Oxford University Press, London, 1963

Backus, G. E., The field lines of an axisymmetric magnetic field, Geophys. J., 93, 413-417, 1988.

Baker, C. P., J. E. Humble, and M. L. Duldig, Effect of the magnetic field model on cosmic ray coupling coefficient calculations, J. Geomagn. Geoelectr., 42, 1137-1144, 1990.

Baker, D. N., T. I. Pulkkinen, R. L. McPherron, J. D. Craven, L. A. Frank, R. D. Elphinstone, J. S. Murphree, J. F. Fennell, R. E. Lopez, and T. Nagai, CDAW 9 analysis of magnetospheric events on May 3, 1986: Event C, J. Geophys. Res., 98, 38153834, 1993.

Baker, K. B., and S. Wing, A new magnetic coordinate system for conjugate studies at high latitudes, J. Geophys. Res., 94, 91399143, 1989.

Baker, K. B., R. A. Greenwald, J. M. Ruohoniemi, J. R. Dudeney, M. Pinnock, N. Mattin, and J. M. Leonard, PACE: Polar Anglo-American Conjugate Experiment, EOS Trans. Am. Geophys. Union, 70, 785, 799, 1989.

Barraclough, D. R., Spherical harmonic analyses of the geomagnetic field for eight epochs between 1600 and 1910, Geophys. J. R. Astron. Soc., 36, 497-513, 1974.

Barraclough, D. R., Modelling the geomagnetic field, J. Geomagn. Geoelectr., 42, 1051-1070, 1990.

Barraclough, D. R., L. D. Williams, and J. M. Quinn, US/UK Candidates for the Definitive Geomagnetic Reference Field Model DGRF-85 and the Predictive International Geomagnetic Reference Field Model IGRF-90, J. Geomagn. Geoelectr., 44, 719-734, 1992.

Birn, J., E. W. Hones, J. D. Craven, L. A. Frank, R. D. Elphinstone, and D. P. Stern, On open and closed field line regions in Tsyganenko's field model and their possible associations with horse collar auroras, J. Geophys. Res., 96, 3811-3817, 1991.

Chapman, S., and J. Bartels, Geomagnetism, Vols I and II, Oxford University Press, London, 1940.

Eather, R. H., The auroral oval - a reevaluation, Rev. Geophys. Space Phys., 11, 155-167, 1973.

Elphinstone, R. D., D. Hearn, J. S. Murphree, and L. L. Cogger, Mapping using the Tsyganenko long magnetospheric model and its relationship to Viking auroral images, J. Geophys. Res., 96, 1467-1480, 1991.

Erdélyi, A., W. Magnus, F. Oberhettinger, and F. G. Tricomi, Higher Transcendental Functions, Volume I, McGraw-Hill, New York, 1953.

Evans, D. S., The observations of a near monoenergetic flux of auroral electrons, J. Geophys, Res., 73, 2315-2323, 1968.

Fairfield, D. H., An evaluation of the Tsyganenko magnetic field model, J. Geophys. Res., 96, 1481-1494, 1991.
Feldstein, Y. I., and Yu. I. Galperin, The auroral luminosity structure in the high-latitude upper atmosphere: its dynamics and relationship to the large-scale structure of the Earth's magnetosphere, Rev. Geophys., 23, 217-275, 1985.

Gorney, D. J., U.S. progress in auroral research: 1983-1986, Rev. Geophys., 25, 555-569, 1987.

Greenwald, R. A., W. Weiss, E. Nielsen, and N. R. Thomson, STARE: A new radar auroral backscatter experiment in northern Scandinavia, Radio Sci., 13, 1021-1039, 1978.

Gustafsson, G., A revised corrected geomagnetic coordinate system, Ark. Geofys., 5, 595-617, 1970.

Gustafsson, G., Corrected geomagnetic coordinates for Epoch 1980, in Magnetospheric Currents, Geophysical Monograph 28, Ed. T. A. Potemra, A. G. U, Washington DC., pp 276-283, 1984.

Gustafsson, G., N. E. Papitashvili, and V. O. Papitashvili, A revised corrected geomagnetic coordinate system for Epochs 1985 and 1990, J. Atmos. Terr. Phys., 54, 1609-1631, 1992.

Hakura, Y., Tables and maps of geomagnetic coordinates corrected by the higher-order spherical harmonic terms, Rep. Ionos. Space Res. Jpn., 19, 121-157, 1965.

Hultqvist, B., The geomagnetic field lines in higher approximation, Ark. Geofys., 3, 63-77, 1958.

Jones, B. W., An Introduction to Modern Algebra, MacMillan, New York, 1975.

Jordan, C. E., Empirical models of the magnetospheric magnetic field, Rev. Geophys., 32, 139-157, 1994.

Langel, R. A., International Geomagnetic Reference Field: the sixth generation, J. Geomagn. Geoelectr., 44, 679-707, 1992.

Langel, R. A., R. H. Estes, and T. J. Sabaka, Uncertainty estimates in geomagnetic field modeling, J. Geophys. Res., 94, 1228112299, 1989.

Langel, R. A., T. J. Sabaka, and R. T. Baldwin, The Geomagnetic Field: $1970-1990$ and the NASA candidate models for DGRF 1985 and IGRF 1990, J. Geomagn. Geoelectr., 44, 745-767, 1992.

Lowes, F. J., Some problems in modelling the main geomagnetic field, J. Geomagn. Geoelectr., 42, 961-971, 1990a.

Lowes, F. J., The limitations of numerical models of the main geomagnetic field, J. Geomagn. Geoelectr., 42, 1071-1078, 1990b.

MATLAB Reference Guide, The Math Works, Natick, Mass., 1992.

Matsushita, S., and W. H. Campbell, Physics of Geomagnetic Phenomena, Vols. I and II, Academic Press, New York, 1967.

Mcllwain, C. E., Direct measurement of particles producing visible auroras, J. Geophys. Res., 65, 2727-2747, 1960.

Mcllwain, C. E., Coordinates for mapping the distribution of magnetically trapped particles, J. Geophys. Res., 66, 3681-3691, 1961.

Meng, C.-I., Electron precipitations and polar auroras, Space Sci. Rev., 22, 223-300, 1978.

Meyers, H., and W. M. Davis, A profile of the geomagnetic model user and abuser, J. Geomagn. Geoelectr., 42, 1079-1085, 1990.

NAG Fortran Library Manual, Mark 13, Vol. 6, Numerical Algorithms Group Limited, Oxford, 1988.

Newell, P. T., C.-I. Meng, and D. A. Hardy, Overview of electron and ion precipitation in the auroral oval, in Auroral Physics, Eds. C.-I. Meng, M. J. Rycroft, and L. A. Frank, Cambridge University Press, Cambridge, pp 85-95, 1991.

Nielsen, E., W. Güttler, E. C. Thomas, C. P. Stewart, T. B. Jones, and A. Hedberg, A new radar auroral backscatter experiment, Nature, 304, 712-714, 1983.

Northrop, T. G., The Adiabatic Motion of Charged Particles, Interscience, Wiley, New York, 1963.

Papitashvili, V. O., N. E. Papitashvili, G. Gustafsson, K. B. Baker, A. Rodger, and L. I. Gromova, A comparison between two corrected geomagnetic coordinate systems at high-latitudes, J. Geomagn, Geoelectr., 44, 1215-1224, 1992.

Peddie, N. W., International Geomagnetic Reference Field: the third generation, J. Geomagn. Geoelectr., 34, 309-326, 1982. 
Pulkkinen, T. I., A study of magnetic field and current configurations in the magnetotail at the time of a substorm onset, Planet. Space Sci., 39, 833-845, 1991.

Pulkkinen, T. I., D. N. Baker, D. H. Fairfield, R. J. Pellinen, J. S. Murphree, R. D. Elphinstone, R. L. McPherron, J. F. Fennell, R. E. Lopez, and T. Nagai, Modeling the growth phase of a substorm using the Tsyganenko model and multi-spacecraft observations: CDAW-9, Geophys. Res. Lett., 18, 1963-1966, 1991.

Pulkkinen, T. I., D. N. Baker, R. J. Pellinen, J. Büchner, H. E. J. Koskinen, R. E. Lopez, R. L. Dyson, and L. A. Frank, Particle scattering and current sheet stability in the geomagnetic tail during the substorm growth phase, J. Geophys. Res., 97, 1928319297, 1992.

Rishbeth, H., and P. J. S. Williams, The EISCAT ionospheric radar: the system and its early results, Q.J.R. Astron. Soc., 26, 478-512, 1985.

Roederer, J. G., Dynamics of Geomagnetically Trapped Radiation, Springer, Berlin, Heidelberg, New York, 1970.

Roederer, J. G., Geomagnetic field distortions and their effects on radiation belt particles, Rev. Geophys. Space Phys., 10, 599-630, 1972.

Schneider, D. A., and D. V. Kent, The time-averaged paleomagnetic field, Rev. Geophys., 28, 71-96, 1990.

Shea, M. A., and D. F. Smart, The influence of the changing geomagnetic field on cosmic ray measurements, J. Geomagn. Geoelectr., 42, 1107-1121, 1990.

Stasiewicz, K., Polar cusp topology and position as a function of interplanetary magnetic field and magnetic activity: comparison of a model with Viking and other observations, J. Geophys. Res., 96, 15789-15800, 1991.

Stassinopoulos, E. G., L. J. Lanzerotti, and T. J. Rosenberg, Temporal variations in the Siple Station conjugate area, $J$. Geophys, Res., 89, 5655-5659, 1984.

Stern, D. P., Representation of magnetic fields in space, Rev. Geophys. Space Phys., 14, 199-214, 1976.

Stern, D. P., A model of the magnetospheric tail with current-free lobes, Planet. Space Sci., 38, 255-261, 1990.

Stern, D. P., A simple model of Birkeland currents, J. Geophys. Res., 98, 5691-5706, 1993

Stern, D. P., The art of mapping the magnetosphere, J. Geophys. Res., 99, 17169-17198, 1994.

Stern, D. P., and N. A. Tsyganenko, Uses and limitations of the Tsyganenko magnetic field models, EOS Trans. Am. Geophys. Union, 73, 489, 493-494, 1992.
Störmer, C., The Polar Aurora, Oxford University Press, London, 1955.

Stuart, W. F., D. R. Barraclough, and J. Preston, Magnetic reference in navigation - the Earth as a magnet, J. Navig., 41, 336-357, 1988.

Thompson, R., and D. R. Barraclough, Geomagnetic secular variation based on spherical harmonic and cross validation analyses of historical and archaeomagnetic data, J. Geomagn. Geoelectr., 34, 245-263, 1982.

Tsyganenko, N. A., Global quantitative models of the geomagnetic field in the cislunar magnetosphere for different disturbance levels, Planet. Space Sci., 35, 1347-1358, 1987.

Tsyganenko, N. A., A magnetospheric magnetic field model with a warped tail current sheet, Planet. Space Sci., 37, 5-20, 1989.

Tsyganenko, N. A., Quantitative models of the magnetospheric magnetic field: methods and results, Space Sci. Rev., 54, 75-186, 1990.

Tsyganenko, N. A., Methods for quantitative modeling of the magnetic field from Birkeland currents, Planet. Space Sci., 39, 641-654, 1991.

Tsyganenko, N. A., A global analytical representation of the magnetic field produced by the Region 2 Birkeland currents and the partial ring current, J. Geophys. Res., 98, 5677-5690, 1993.

Vallarta, M. S., Theory of the geomagnetic effects of cosmic radiation, in Encyclopedia of Physics, 46/1, Ed. S. Flügge, Springer, Berlin, Heidelberg, New York, pp 88-129, 1961.

Walt, M., Introduction to Geomagnetically Trapped Radiation, Cambridge University Press, Cambridge, 1994.

Weeks, R. J., M. Fuller, and I.. Williams, A model for transitional field geometries involving low-order zonals and drifting nondipole harmonics, J. Geophys, Res., 93, 11613-11620, 1988.

Williams, I., and M. Fuller, Zonal harmonic models of reversal transition fields, J. Geophys. Res., 86, 11657-11665, 1981.

Willis, D. M., and L. R. Young, Equation for the field lines of an axisymmetric magnetic multipole, Geophys. J. R. Astron. Soc., 89, 1011-1022, 1987.

Willis, D. M., J. R. Singh, and K. S. C. Freeman, Uncertainties in field-line tracing in the magnetosphere. Part II: the complete: internal geomagnetic field, Ann. Geophysicae, submitted, 1997.

Winch, D. E., The fourth-order geomagnetic multipole: the sedecimupole, Pure Appl. Geophys., 67, 112-122, 1967.

Wolfram, S., Mathematica: A System for Doing Mathematics by Computer, Addison-Wesley, Redwood City, Calif., 1988. 